\title{
An efficient Wave Based Method for 2D acoustic problems containing corner singularities
}

\author{
Elke Deckers, Bart Bergen, Bert Van Genechten, Dirk Vandepitte, Wim Desmet \\ Department of Mechanical Engineering, Katholieke Universiteit Leuven, \\ Celestijnenlaan 300B, 3001 Heverlee, Belgium
}

\begin{abstract}
The Wave Based Method is an efficient alternative prediction technique based on a Trefftz approach which, as compared to element-based methods, can be applied in a wider frequency range at a reasonable cost. This paper discusses the use of this method for the particular case of $2 \mathrm{D}$ acoustic problems where singularities are present in the corners of the acoustic domain. The conventional set of expansion functions used to approximate the dynamic pressure field is extended with special purpose functions that incorporate the singular behaviour in the region around the singular corners. This enrichment comes with a beneficial convergence rate while only a small increase of computational effort is incurred. This is illustrated for various problem settings, including different combinations of both homogeneous and non-homogeneous boundary conditions and bounded as well as unbounded problems are considered.
\end{abstract}

Keywords: Helmholtz equation, 2D acoustics, Corner singularities, Wave Based Method, Trefftz

\section{Introduction}

Over the last decades, numerical prediction techniques for acoustic analysis have gained importance to take into account sound comfort and sound emission of a product in the design and development process. This way, the use of physical prototypes can be restricted to a minimum, but it requires efficient and accurate predictions. However, for most numerical prediction techniques it is difficult to obtain accurate solutions when singularities are present in the problem domain. For a polygonal acoustic domain, singularities can exist at corner points and even at points on flat boundaries where boundary conditions change. In standard numerical approaches the approximation field is generally not suitable to represent the steep gradient in the near vicinity of the singular corner. Measures have to be taken, otherwise the convergence rate is affected. This paper focuses on singularities in two-dimensional, both bounded and unbounded, acoustic problems containing a single acoustic fluid.

It is well-known that standard element based procedures such as the Finite Element Method (FEM) [1] and the Boundary Element Method (BEM) [2] lose accuracy when corner singularities are present. As a result many specially

\footnotetext{
*Corresponding author; Tel.:+32 163224 80; fax: +32 163229 87; http://www.mech.kuleuven.be/ E-mail address: Elke.Deckers@mech.kuleuven.be

Email address: Elke.Deckers@mech.kuleuven. be (Elke Deckers) 
adapted numerical methods have been developed to efficiently tackle this type of problems. An overview of singularity treatments of elliptic boundary value problems is given by $\mathrm{Li}$ and $\mathrm{Lu}$ [3]. A first approach is to study graded meshes near the singularity within the FEM, for instance see $[4,5,6]$ and references therein, as well as within the BEM [7].

In the category of FE methods, some other approaches can be found. Special singular basis functions can be applied in the region around the singularity $[8,9]$. Wu and Han [10] introduce an artificial boundary and reduce the original problem to a boundary value problem away from the singularity. Lucas and Oh [11] apply the method of auxiliary mapping and implicitly create singular basis functions by locally transforming a region around each singularity to a new domain. Some other improvements to the FEM to tackle singularities include the use of the DtN FEM [12], and the coupling of the p-FEM with spectral methods [13].

Regarding adaptations to the BEM, Chen et al. $[14,15]$ use the dual BEM to deal with the Helmholtz equation in domains with singularities. The dual BEM utilises the singular and hypersingular equation at the same time. Marin et al. [16] modify the original problem by a change of variables and take into account the effect of the singular solution on the boundary conditions. Extra conditions are imposed near the singular corner.

Apart from element-based techniques and all the methods derived from their concepts (for instance stabilised [17] and multiscale [18, 19] methods), also Trefftz methods [20] are applied for acoustic simulations. Trefftz methods apply different shape and weighting functions and use exact solutions of the governing equations to approximate the field variables [21, 22]. The incorporation of a priori knowledge of the solution field leads generally to smaller model sizes and a faster convergence.

Nevertheless also the accuracy of Trefftz methods is adversely affected when singularities are present. For example Huttunen et al. [23] and Gamallo et al. [24] compare the ultra-weak variational formulation (UWVF) [25] with respectively the partition of unity FEM (PUFEM) and the least squares method (LSM) for the solution of a singular L-shaped domain and report only a moderate performance ( $0.4 \%$ accuracy) and suggest progressive refinement of the mesh near the singularity or the use of alternative basis functions.

$\mathrm{Li}$ [26] gives an overview of the existing methods to tackle the Helmholtz equation and focuses on Trefftz methods. He applies the Boundary Approximation Method (BAM) which uses piecewise particular solutions. Exact solutions of the singularity are embedded and excellent accuracies are obtained for the singular Motz's problem. Antunes et al. [27] enrich the approximation set of the Method of Fundamental Solutions (MFS) with corner adapted shape functions to obtain an improved convergence. Marin [28] applies a singularity subtraction technique to the MFS, similar as in [16] to reformulate the problem. Stojek [29] applies least-squares T-elements for the Helmholtz equation in an unbounded domain and adds special purpose functions in the vicinity of singular corners, fulfilling the Neumann homogeneous boundary conditions and applies a multipole expansion for the scattered field. He reports that by adding the singular functions, the convergence accelerates and becomes of the same type as in the case of regular problems without a singularity. Barnett and Betcke [30] divide unbounded problem domains in subdomains containing maximum one corner and apply fractional order Fourrier-Bessel functions at the corners to match the asymptotic behaviour and approximate the scattered field with the MFS. The method can be applied for sound hard and sound soft scatterers. 
In this paper the Wave Based Method (WBM) [31] is studied for 2D acoustic problems with singularities. The WBM is an alternative deterministic technique which is also based on the Trefftz principle. The problem domain is partitioned into a small number of convex subdomains. The dynamic response variables within each subdomain are described using so-called wave functions which exactly satisfy the homogeneous part of the governing differential equation(s) and particular solutions are added to comply with the non-homogeneous part. In this way, the wave functions may only violate the imposed boundary and continuity conditions on the interfaces. Enforcing the associated residual errors to zero in a weighted residual scheme yields a system of algebraic equations which can be solved for the contribution factors of each wave function in the expansion set of the dynamic field variables. The WBM has been successfully applied to many steady-state interior [21, 32] and exterior [33, 34, 35] acoustic problems, but also for structural [36, 37] and poroelastic [38, 39] problems. Comparisons with element-based approaches are provided in each of these publications. The method has furthermore been combined in a hybrid way with the FEM [40] and the Statistical Energy Analysis (SEA) [41] for more complex vibro-acoustic problems. For both structural [37] and poroelastic [39] WB models it was shown that the accuracy deteriorates when stress singularities exist in corners of the calculation domain. By adding special purpose functions which exactly incorporate the singular behaviour, convergence problems were remedied. This paper investigates if a similar strategy can be applied to improve the convergence of the WBM in the case of acoustic problems with corner singularities.

The paper is organised as follows. Section 2 shortly reviews the acoustic problem definition for both bounded and unbounded 2D acoustic problems. The next section explains the Wave Based Method. Section 4 considers the existence of singularities in more detail and describes functions which exactly represent the singular behaviour in the near vicinity of a corner and therefore can be used as special purpose enrichment functions within the WBM. Finally, the use of the WBM is demonstrated for three different validation examples including different types of corner singularities. It is shown that the incorporation of the enrichment functions leads to an improved efficiency.

\section{Problem description}

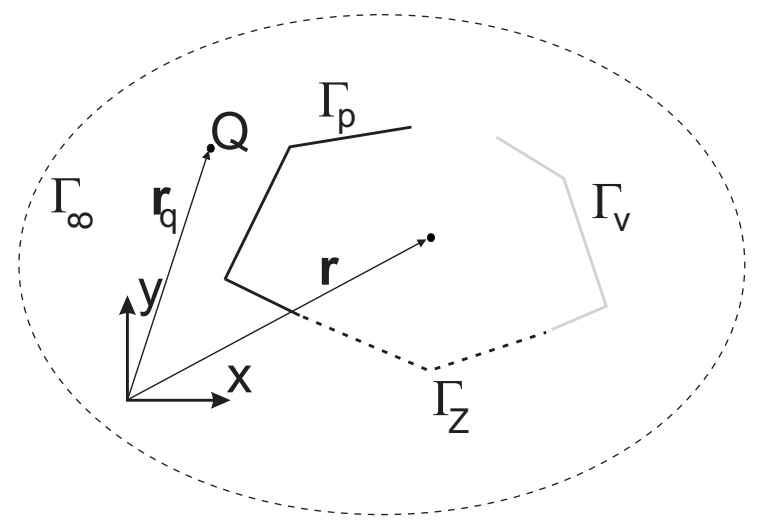

Figure 1: General 2D unbounded acoustic problem. 
The problem considered in this paper is a general two-dimensional steady-state acoustic problem, which can either be bounded or unbounded as illustrated by Figure 1. The fluid is characterised by its density $\rho$ and speed of sound $c$. The steady-state acoustic pressure inside the problem domain is governed by the inhomogeneous Helmholtz equation [42]:

$$
\nabla^{2} p(\boldsymbol{r})+k^{2} p(\boldsymbol{r})=-j \rho \omega \delta\left(\boldsymbol{r}, \boldsymbol{r}_{\boldsymbol{q}}\right) q
$$

with $\boldsymbol{r}=(x, y)$ the location vector, defining a point in the two-dimensional space, $\nabla^{2} \bullet=\frac{\partial^{2} \bullet}{\partial x^{2}}+\frac{\partial^{2} \bullet}{\partial y^{2}}$ the Laplace operator, $\delta$ the Dirac-delta function, $\omega$ the circular frequency, $k=\omega / c$ the acoustic wave number and $j=\sqrt{-1}$ the imaginary unit. The fluid is excited by an acoustic volume velocity source with source strength $q$. Throughout this article, a harmonic time dependence $e^{j \omega t}$ of the dynamic quantities and excitations is assumed. The problem boundary $\Gamma$ consists of two parts in case the problem domain is unbounded: the finite part of the boundary $\Gamma_{b}$ and the boundary at infinity $\Gamma_{\infty}$. For a bounded domain, obviously, only $\Gamma_{b}$ has to be considered.

The finite part of the boundary can be further divided in three non-overlapping parts: $\Gamma_{b}=\Gamma_{v} \cup \Gamma_{p} \cup \Gamma_{Z}$, where the following boundary condition residuals apply:

$$
\begin{array}{ll}
\boldsymbol{r} \in \Gamma_{v}: & R_{v}=\mathcal{L}_{v}(p(\boldsymbol{r}))-\bar{v}_{n}(\boldsymbol{r})=0, \\
\boldsymbol{r} \in \Gamma_{p}: & R_{p}=p(\boldsymbol{r})-\bar{p}(\boldsymbol{r})=0, \\
\boldsymbol{r} \in \Gamma_{Z}: & R_{Z}=\mathcal{L}_{v}(p(\boldsymbol{r}))-\frac{p(\boldsymbol{r})}{\bar{Z}_{n}(\boldsymbol{r})}=0,
\end{array}
$$

where the quantities $\bar{v}_{n}, \bar{p}$ and $\bar{Z}_{n}$ are, respectively, the imposed normal velocity, pressure and normal impedance. $n$ is the local normal on the boundary and the velocity operator $\mathcal{L}_{v}(\bullet)$ is defined as:

$$
\mathcal{L}_{v}(\bullet)=\frac{j}{\rho \omega} \frac{\partial \bullet}{\partial n} .
$$

At the boundary at infinity $\Gamma_{\infty}$ the Sommerfeld radiation condition [43] must be satisfied in order to ensure that all acoustic waves propagate freely towards infinity and that no reflections occur at this boundary,

$$
\lim _{|\boldsymbol{r}| \rightarrow \infty}\left(\sqrt{|\boldsymbol{r}|}\left(\frac{\partial p(\boldsymbol{r})}{\partial|\boldsymbol{r}|}+j k p(\boldsymbol{r})\right)\right)=0 .
$$

The Helmholtz equation together with the associated boundary conditions, define a unique pressure field $p(\boldsymbol{r})$. Once this pressure field is calculated, the derived acoustic quantities such as the acoustic velocity, the intensity and the acoustic power can be obtained.

\section{The Wave Based Method for $2 \mathrm{D}$ acoustics}

The WBM [31] is a numerical prediction technique which is based on an indirect Trefftz approach. It expresses the field variables as an expansion of wave functions which inherently satisfy the governing equation, in this case the Helmholtz equation (1). The degrees of freedom are the weighting factors of the wave functions in this expansion. 
Since the wave functions are exact solutions of the Helmholtz equation, they only violate the boundary and interface conditions. These conditions are enforced using a weighted residual formulation which yields a system of linear equations whose solution vector contains the wave function weighting factors. This section briefly reviews the method for both $2 \mathrm{D}$ bounded and unbounded problems. For a more profound description, the reader is referred to literature $[21,31,33,35,44]$.

The general modelling procedure consists of the following four steps [44], which will be discussed afterwards:

A. partitioning into subdomains;

B. selection of the wave functions in the pressure expansion within each subdomain;

C. construction of the system of equations via a weighted residual formulation of the boundary conditions and the continuity conditions;

D. solution of the system of equations and postprocessing of the dynamic variables.

\subsection{Partitioning into subdomains}

In the case of bounded problems, when the considered problem domain is non-convex, it is, in a first step, partitioned into a number of convex subdomains [31]. When applied to unbounded problems, an initial partitioning of the unbounded domain into a bounded and an unbounded region by a truncation curve $\Gamma_{t}$ precedes the partitioning into convex subdomains [21]. Figure 2 illustrates the principle. The unbounded region exterior to $\Gamma_{t}$ is considered as one acoustic subdomain.

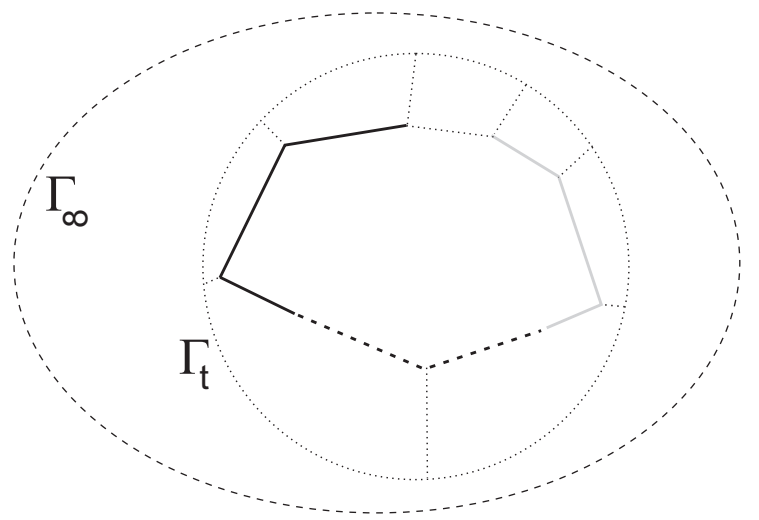

Figure 2: A WB partitioning of the 2D unbounded problem. Subdomain interfaces are shown in dotted line $(\cdots)$.

This way, the total problem domain $\Omega$ is subdivided into $N_{\Omega}$ non-overlapping subdomains $\Omega^{(\alpha)}\left(\alpha=1, \ldots, N_{\Omega}\right)$, which may be bounded or unbounded. On the interface between two subdomains $\Omega^{(\alpha)}$ and $\Omega^{(\beta)}$ continuity conditions need to be imposed. Two different types of approaches can be followed here: directly enforce pressure continuity on one subdomain and normal velocity continuity on the other subdomain or apply impedance continuity conditions. Pluymers [44] has shown that the impedance coupling approach introduces damping into the numerical model which 
has a beneficial effect on the WBM's efficiency. For this reason, only the impedance coupling approach is discussed and applied within this paper. The residuals can then be written as:

$$
\boldsymbol{r} \in \Gamma_{I}: R_{I}^{(\alpha, \beta)}=\left(\frac{j}{\rho \omega} \frac{\partial p^{(\alpha)}(\boldsymbol{r})}{\partial n^{(\alpha)}}-\frac{p^{(\alpha)}(\boldsymbol{r})}{\bar{Z}_{\text {int }}}\right)+\left(\frac{j}{\rho \omega} \frac{\partial p^{(\beta)}(\boldsymbol{r})}{\partial n^{(\beta)}}+\frac{p^{(\beta)}(\boldsymbol{r})}{\bar{Z}_{\text {int }}}\right),
$$

with $n^{(\alpha)}$ the local normal on the subdomain interface $\Gamma_{I}$, outwards of domain $\Omega^{(\alpha)}$ and $\bar{Z}_{\text {int }}$ an impedance coupling factor, typically chosen as $\rho c$ [44]. This continuity condition is enforced on each subdomain, for each interface, yielding two continuity conditions over each subdomain interface.

\subsection{Acoustic pressure expansion}

The steady-state acoustic pressure field $p^{(\alpha)}(\boldsymbol{r})$ in an acoustic subdomain $\Omega^{(\alpha)}$ is approximated by a solution expansion $\hat{p}^{(\alpha)}(\boldsymbol{r})$ :

$$
\begin{aligned}
p^{(\alpha)}(\boldsymbol{r}) \simeq \hat{p}^{(\alpha)}(\boldsymbol{r}) & =\sum_{w=1}^{n_{w}^{(\alpha)}} p_{w}^{(\alpha)} \Phi_{w}^{(\alpha)}(\boldsymbol{r})+\hat{p}_{q}(\boldsymbol{r}) \\
& =\boldsymbol{\Phi}^{(\alpha)}(\boldsymbol{r}) \boldsymbol{p}^{(\alpha)}+\hat{p}_{q}(\boldsymbol{r}) .
\end{aligned}
$$

The wave function contributions $p_{w}^{(\alpha)}$ are the weighting factors for each of the selected wave functions $\Phi_{w}^{(\alpha)}$. Together they form the vector of degrees of freedom $\boldsymbol{p}^{(\alpha)}$. The corresponding a priori defined wave functions are collected in the row vector $\boldsymbol{\Phi}^{(\alpha)}$. The term $\hat{p}_{q}(\boldsymbol{r})$ represents a particular solution resulting from acoustic source terms $q$ in the right hand side of the inhomogeneous Helmholtz equation (1). The set of all $n_{W}=\sum_{\alpha=1}^{N_{\Omega}} n_{w}^{(\alpha)}$ acoustic wave function contributions $p_{w}^{(\alpha)}$ is collected in the column vector $\boldsymbol{p}$, while the row vector $\boldsymbol{\Phi}$ contains all $n_{W}$ wave functions.

\subsubsection{Wave functions for a bounded subdomain}

Each acoustic wave function $\Phi_{w}^{(\alpha)}(\boldsymbol{r})$ in solution expansion (8) exactly satisfies the homogeneous part of the Helmholtz equation (1). For 2D bounded subdomains, two types of wave functions are distinguished, the so-called rand s-set:

$$
\sum_{w=1}^{n_{w}^{(\alpha)}} p_{w}^{(\alpha)} \Phi_{w}^{(\alpha)}(\boldsymbol{r})=\sum_{w_{r}=1}^{n_{w r}^{(\alpha)}} p_{w_{r}}^{(\alpha)} \Phi_{w_{r}}^{(\alpha)}(\boldsymbol{r})+\sum_{w_{s}=1}^{n_{w s}^{(\alpha)}} p_{w_{s}}^{(\alpha)} \Phi_{w_{s}}^{(\alpha)}(\boldsymbol{r}),
$$

with the total number of wave functions in subdomain $\Omega^{(\alpha)}, n_{w}^{(\alpha)}$, defined as:

$$
n_{w}^{(\alpha)}:=n_{w_{r}}^{(\alpha)}+n_{w_{s}}^{(\alpha)}
$$

The wave functions are defined as:

$$
\Phi_{w}^{(\alpha)}(\boldsymbol{r}(x, y))=\left\{\begin{array}{l}
\Phi_{w_{r}}^{(\alpha)}(x, y)=\cos \left(k_{x w_{r}}^{(\alpha)} x\right) e^{-j k_{y w_{r}} y} \\
\Phi_{w_{s}}^{(\alpha)}(x, y)=e^{-j k_{x w_{s}}^{(\alpha)} x} \cos \left(k_{y w_{s}}^{(\alpha)} y\right)
\end{array} .\right.
$$

The only requirement for the wave functions (11) to be exact solutions of the homogeneous part of the Helmholtz equation (1) is that the wave number components have to satisfy the associated dispersion relation:

$$
\left(k_{x w_{r}}^{(\alpha)}\right)^{2}+\left(k_{y w_{r}}^{(\alpha)}\right)^{2}=\left(k_{x w_{s}}^{(\alpha)}\right)^{2}+\left(k_{y w_{s}}^{(\alpha)}\right)^{2}=k^{2}
$$


As a result, an infinite number of wave functions (11) can be defined for expansion (8). Desmet [31] proposes to select the following wave number components:

$$
\begin{aligned}
& \left(k_{x w_{r}}^{(\alpha)}, k_{y w_{r}}^{(\alpha)}\right)=\left(\frac{w_{1}^{(\alpha)} \pi}{L_{x}^{(\alpha)}}, \pm \sqrt{k^{2}-\left(k_{x w_{r}}^{(\alpha)}\right)^{2}}\right), \\
& \left(k_{x w_{s}}^{(\alpha)}, k_{y w_{s}}^{(\alpha)}\right)=\left( \pm \sqrt{k^{2}-\left(k_{y w_{s}}^{(\alpha)}\right)^{2}}, \frac{w_{2}^{(\alpha)} \pi}{L_{y}^{(\alpha)}}\right)
\end{aligned}
$$

with $w_{1}^{(\alpha)}=0,1,2, \ldots, w_{1, \max }^{(\alpha)}$ and $w_{2}^{(\alpha)}=0,1,2, \ldots, w_{2, \max }^{(\alpha)}$. The dimensions $L_{x}^{(\alpha)}$ and $L_{y}^{(\alpha)}$ represent the dimensions of the (smallest) bounding rectangle, circumscribing the considered subdomain $\Omega^{(\alpha)}$. With this selection for the wave numbers, the wave functions (11) represent a standing wave along one coordinate direction of the bounding box, multiplied with a propagating or evanescent component along the other direction.

The number $n_{w}^{(\alpha)}$ of wave functions for each subdomain $\Omega^{(\alpha)}$ is determined from the user-defined truncation factor $N$ by

$$
\begin{aligned}
w_{1, \text { max }}^{(\alpha)}: & =\max \left\{w_{1}^{(\alpha)} \in \mathbb{N} \mid \frac{w_{1}^{(\alpha)} \pi}{L_{x}^{(\alpha)}}<N k\right\}, \\
w_{2, \text { max }}^{(\alpha)}: & =\max \left\{w_{2}^{(\alpha)} \in \mathbb{N} \mid \frac{w_{2}^{(\alpha)} \pi}{L_{y}^{(\alpha)}}<N k\right\}, \\
& n_{w_{r}}^{(\alpha)}:=2\left(w_{1, \text { max }}^{(\alpha)}+1\right), \\
& n_{w_{s}}^{(\alpha)}:=2\left(w_{2, \text { max }}^{(\alpha)}+1\right),
\end{aligned}
$$

and (10). The adopted truncation rule is frequency dependent and selects the number of wave functions such that the highest oscillatory wave number component of the $r$ - and the s-set is approximately equal to a user-defined truncation factor $N$ times the physical wavenumber $k$ of the considered problem. This truncation rule (14) is applied to all bounded subdomains such that the approximation field in adjacent subdomains exhibits a similar spatial variation along the interface. This way, a homogeneous approximation resolution is obtained throughout the problem domain. The number of wave functions increases approximately linearly with frequency. Numerical experiments have shown that for typical acoustic applications a choice of $N=1, \ldots, 6$ gives a good balance between the computational cost of the WBM models and their prediction accuracy [32, 40, 44, 45].

The particular solution $\hat{p}_{q}(\boldsymbol{r})$ in equation (8) represents the free field pressure field due to an acoustic point source at position $\left(x_{q}, y_{q}\right)$ :

$$
\hat{p}_{q}(x, y)=\frac{\rho \omega q}{4} H_{0}^{(2)}\left(k r_{q}\right)
$$

with $r_{q}=\sqrt{\left(x-x_{q}\right)^{2}+\left(y-y_{q}\right)^{2}}$ and $H_{0}^{(2)}(\bullet)$ the zero-order Hankel function of the second kind.

\subsubsection{Wave functions for an unbounded subdomain}

The wave functions for the unbounded domains are chosen to implicitly comply with not only the Helmholtz equation, but also with the Sommerfeld radiation condition (6) at $\Gamma_{\infty}$. This removes the need to explicitly impose a radiation condition, similar as in the BEM. Herrera [46] shows that the following expansion $\hat{p}_{e}$, exterior to a circular truncation curve with radius $R_{t}$, yields a convergent set for the pressure field $p_{e}$, defined by a Neumann condition on 
the infinitely long cylinder with radius $R_{t}$ :

$$
\begin{aligned}
p_{e}(r, \theta) \simeq \hat{p}_{e}(r, \theta) & =p_{e, c 0} H_{0}^{(2)}(k r) \\
& +\sum_{n=1}^{n_{u}}\left(p_{e, c n} H_{n}^{(2)}(k r) \cos (n \theta)+p_{e, s n} H_{n}^{(2)}(k r) \sin (n \theta)\right),
\end{aligned}
$$

with $r$ and $\theta$ polar coordinates and $H_{n}^{(2)}(\bullet)$ the $n$th order Hankel function of the second kind. The contributions $p_{e, c 0}$, $p_{e, c n}$ and $p_{e, s n}$ are determined by the velocity boundary condition. From this expansion, the following wave function set for unbounded domains exterior to a circular truncation curve with radius $R_{t}$ is derived [35, 44]:

$$
\Phi_{w}^{(u b)}(r, \theta)=\left\{\begin{array}{c}
\Phi_{w_{c}}^{(u b)}(r, \theta)=H_{w_{c}}^{(2)}(k r) \cos \left(w_{c} \theta\right) \\
\Phi_{w_{s}}^{(u b)}(r, \theta)=H_{w_{s}}^{(2)}(k r) \sin \left(w_{s} \theta\right)
\end{array},\right.
$$

with $w_{c}=0,1,2, \ldots, w_{c, \max }$ and $w_{s}=1,2,3, \ldots, w_{s, \max }$. As for bounded domains, the series of functions (18) is truncated to $n_{w}^{(u b)}$ functions, in order to be used in a numerical scheme. A truncation rule similar to equation (14) for the bounded domains determines the highest orders $w_{c, \max }$ and $w_{s, \max }$ of the Hankel functions used in the exterior wave function expansion [45]:

This truncation yields a total of

$$
\begin{aligned}
& w_{c, \text { max }}:=\max \left\{w_{c} \in \mathbb{N} \mid \frac{w_{c}}{2 R_{t}}<N k\right\}, \\
& w_{s, \text { max }}:=\max \left\{w_{s} \in \mathbb{N} \mid \frac{w_{s}}{2 R_{t}}<N k\right\} .
\end{aligned}
$$

$$
n_{w}^{(u b)}:=w_{c, \max }+w_{s, \max }+1
$$

wave functions in the unbounded domain. The physical interpretation of this rule is that a desired circumferential resolution of $\lambda /(2 N)$ is approximately obtained on the truncation $\Gamma_{t}$, with $\lambda=2 \pi / k$ the physical wavelength. Using this rule-of-thumb ensures a similar resolution of the functions in the unbounded and bounded subdomains, as can be seen by comparing equations (14) and (19). The functions (18) represent a harmonic distribution of the amplitude on the truncation, multiplied with the Hankel function $H_{n}^{(2)}(k r)$, describing the radial decay of an outgoing acoustic wave.

The particular solution for a point source (16) can also be applied for unbounded domains. For unbounded problems another commonly used excitation for scattering problems is a plane wave source. For this source, the particular term yields:

$$
\hat{p}_{q}(\boldsymbol{r})=Q_{p w} e^{j \boldsymbol{k} \cdot \boldsymbol{r}},
$$

with $Q_{p w}$ the plane wave amplitude, $k=\left(k_{x}, k_{y}\right)=(k \cos \phi, k \sin \phi)$ the wave vector and $\phi$ the propagation angle.

\subsection{Acoustic wave model}

The proposed expansion functions (11) and (18) exactly satisfy the Helmholtz equation (1) inside the domain and the wave functions for unbounded domains also fulfil the Sommerfeld radiation condition (6) at infinity. The boundary conditions (2) and subdomain continuity (7) are enforced through a weighted residual formulation. 
For each subdomain, the residuals are orthogonalised with respect to a weighting function $\tilde{p}^{(\alpha)}(\boldsymbol{r})$ or its derivative. The weighted residual formulation, applying the introduced residual functions, is expressed as:

$$
\begin{aligned}
& \int_{\Gamma_{v}^{(\alpha)}} \tilde{p}^{(\alpha)}(\boldsymbol{r}) R_{v}^{(\alpha)}(\boldsymbol{r}) \mathrm{d} \Gamma+\int_{\Gamma_{Z}^{(\alpha)}} \tilde{p}^{(\alpha)}(\boldsymbol{r}) R_{Z}^{(\alpha)}(\boldsymbol{r}) \mathrm{d} \Gamma \\
& -\int_{\Gamma_{p}^{(\alpha)}} \mathcal{L}_{v}^{(\alpha)}\left(\tilde{p}^{(\alpha)}(\boldsymbol{r})\right) R_{p}^{(\alpha)}(\boldsymbol{r}) d \Gamma+\sum_{\beta=1, \beta \neq \alpha}^{N_{\Omega}} \int_{\Gamma_{I}^{(\alpha, \beta)}} \tilde{p}^{(\alpha)}(\boldsymbol{r}) R_{I}^{(\alpha, \beta)}(\boldsymbol{r}) \mathrm{d} \Gamma=0 .
\end{aligned}
$$

Like in the Galerkin weighting procedure, used in the FEM, the weighting functions $\tilde{p}^{(\alpha)}$ are expanded in terms of the same set of acoustic wave functions used in the pressure expansions (8) and (18):

$$
\tilde{p}^{(\alpha)}(\boldsymbol{r})=\sum_{w=1}^{n_{w}^{(\alpha)}} \tilde{p}_{w}^{(\alpha)} \Phi_{w}^{(\alpha)}(\boldsymbol{r})=\boldsymbol{\Phi}^{(\alpha)}(\boldsymbol{r}) \tilde{\boldsymbol{p}}^{(\alpha)} .
$$

The weighted residual formulation applying pressure and velocity continuity conditions instead of the impedance coupling conditions (7) is given in [44]. Substitution of the pressure expansions (8) and (18) and the weighting function expansion (23) for subdomain $\Omega^{(\alpha)}$ into the weighted residual formulation (22) yields a set of $n_{w}^{(\alpha)}$ linear equations in the $n_{W}$ unknown wave function contribution factors. One such matrix equation is obtained for each subdomain. Combination of the $N_{\Omega}$ systems yields the acoustic WB model, consisting of $n_{W}$ algebraic equations in the $n_{W}$ unknown wave function contribution factors:

$$
\left[\boldsymbol{A}_{\boldsymbol{a} a}\right]\{\boldsymbol{p}\}=\{\boldsymbol{b}\}
$$

For a detailed description of the system matrices, the reader is referred to literature $[31,32,33]$. Due to the oscillatory behaviour of the wave functions, care should be taken when evaluating the integrals in (22). Standard Gaussian quadrature has proven to be adequate [47], provided sufficient integration points are used. For all calculations 12 integration points per wavelength (of the wave functions) are used.

\subsection{Solution and postprocessing}

The resulting model (24) can be solved for the unknown wave function contributions $\boldsymbol{p}$. The final step in the modelling process is back substitution of these contribution factors into the pressure expansions (8), yielding an analytical description of the approximated dynamic pressure field $\hat{p}(\boldsymbol{r})$. Also derived quantities such as acoustic velocities or intensities can be obtained.

\section{Singularities}

This paper focuses on the existence and treatment of acoustic singularities. For a polygonal acoustic domain, singularities can exist at corner points and even at points on flat boundaries where boundary conditions change. Typically, this kind of singularity arises when the internal angle formed by the two sides of a corner exceeds a critical value, which depends on the imposed boundary conditions. It was indicated by Vanmaele et al. [37] for plate bending 
applications and Deckers et al. [39] for poroelastic applications, that the WBM suffers from convergence problems when singularities are present. These problems originate from the global character of the wave functions, which have difficulties capturing the singular behaviour in the corner. Problems of the same kind are expected when singularities are present in corners of acoustic problem domains.

This section commences with a review of analytical solutions that accurately describe the pressure field and the velocity field in the vicinity of a corner point in case homogeneous boundary conditions are applied. This allows to predict if corner singularities are present and to define functions that accurately represent the singular behaviour in the corner. Afterwards, non-homogeneous boundary conditions are discussed for which generally no analytical solutions can be found. A parallel is drawn between dynamic and static solutions for the infinite wedge domain considering the same boundary conditions. These static solutions have the same asymptotic behaviour near the corner point and they can be used to verify if non-homogeneous boundary conditions lead to additional singularities. Also the case of Robin boundary conditions is discussed. Section 4.5 explains how enrichment functions can be applied within the WBM. A similar approach as in $[37,39]$ is followed here. If singularities are present, the set of wave functions is extended with additional enrichment functions which accurately represent the singular behaviour in the corner point, to enhance the convergence behaviour of the WBM.

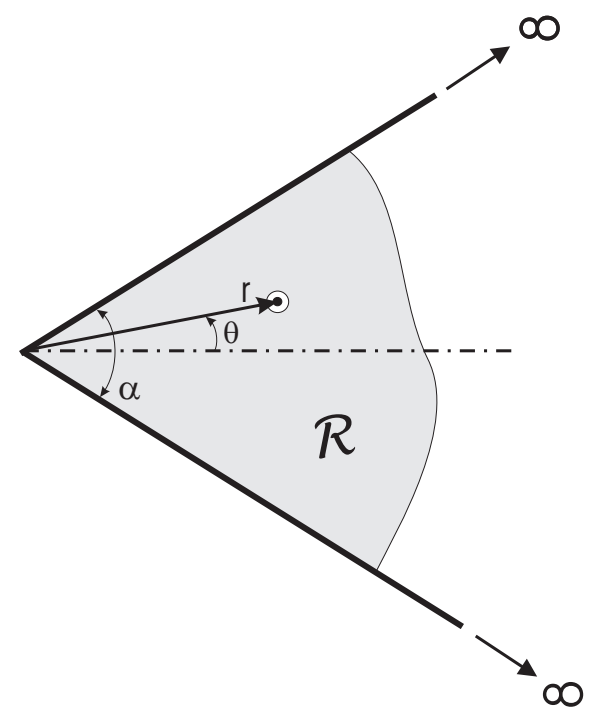

Figure 3: 2D acoustic wedge domain.

\subsection{Analytical dynamic solution for an infinite wedge domain}

The analytical solution of an infinite acoustic wedge has been widely studied in literature and only the main results will be reviewed here. The reader is referred to Appendix A for a more complete discussion.

The analytical solution for the infinite wedge has to satisfy: 

A. the homogeneous Helmholtz equation;
B. the applied boundary conditions;
C. the regularity conditions at the corner tip, $|p(0, \theta)|<\infty$.

As shown in Figure 3, polar cylindrical coordinates $r$ and $\theta$ are employed and the open angular region of interest $\mathcal{R}$ with an internal angle $\alpha$ is given by:

$$
\mathcal{R}=\left\{(r, \theta) \mid 0 \leq r<\infty,-\frac{\alpha}{2} \leq \theta \leq \frac{\alpha}{2}\right\} .
$$

As described in Appendix A, by assuming a separable function for the pressure field $p(r, \theta)$ in polar coordinates and applying the regularity conditions, the following admissible pressure fields are found:

$$
p(r, \theta)=\sum_{b=1}^{\infty}\left[A_{\lambda_{b}} \cos \left(\lambda_{b} \theta\right) J_{\lambda_{b}}(k r)+A_{\lambda_{b}^{*}} \sin \left(\lambda_{b}^{*} \theta\right) J_{\lambda_{b}^{*}}(k r)\right],
$$

with $J_{\lambda}(z)$ the ordinary Bessel functions of the first kind and $\lambda=\lambda_{b}$ or $\lambda_{b}^{*}$ the eigenvalues. The eigenvalues $\lambda_{b}$ are associated with symmetric eigenfunctions with respect to the axis $\theta=0$ and the eigenvalues $\lambda_{b}^{*}$ are associated with anti-symmetric eigenfunctions. The accompanying velocity fields are:

$$
\begin{aligned}
& v_{r}(r, \theta)=\frac{j}{\rho \omega} \sum_{b=1}^{\infty}\left[\frac{A_{\lambda_{b}} k}{2} \cos \left(\lambda_{b} \theta\right)\left(J_{\lambda_{b}-1}(k r)-J_{\lambda_{b}+1}(k r)\right)+\frac{A_{\lambda_{b}^{*}} k}{2} \sin \left(\lambda_{b}^{*} \theta\right)\left(J_{\lambda_{b}^{*}-1}(k r)-J_{\lambda_{b}^{*}+1}(k r)\right)\right], \\
& v_{\theta}(r, \theta)=\frac{j}{\rho \omega} \frac{1}{r} \sum_{b=1}^{\infty}\left[-A_{\lambda_{b}} \lambda_{b} \sin \left(\lambda_{b} \theta\right) J_{\lambda_{b}}(k r)+A_{\lambda_{b}^{*}} \lambda_{b}^{*} \cos \left(\lambda_{b}^{*} \theta\right) J_{\lambda_{b}^{*}}(k r)\right] .
\end{aligned}
$$

The velocity fields tend to infinity at $r \rightarrow 0$ if $0<\lambda<1$. In this case, a singularity can exist in the velocity field and the order of the singularity is $\lambda-1$. The eigenvalues $\lambda$ depend on $\alpha$ and the applied boundary conditions on both sides of the wedge.

\subsection{Analytical solution for various homogeneous boundary conditions}

This section overviews solutions of the characteristic equation for different combinations of homogeneous boundary conditions and concludes for each of the combinations of boundary conditions when singularities can be present in the velocity field. A similar overview (yet in a shifted coordinate system) is given in [16]:

- Dirichlet-Dirichlet boundary conditions: $p\left(r,-\frac{\alpha}{2}\right)=p\left(r, \frac{\alpha}{2}\right)=0$;

$$
\begin{array}{ll}
\lambda_{b}=(2 b-1) \frac{\pi}{\alpha}, & \text { with } b=1,2, \ldots, \\
\lambda_{b}^{*}=2 b \frac{\pi}{\alpha}, & \text { with } b=1,2, \ldots .
\end{array}
$$

Singularities can be present if $\alpha>\pi$ for the symmetric set.

- Neumann-Neumann boundary conditions: $v_{\theta}\left(r,-\frac{\alpha}{2}\right)=v_{\theta}\left(r, \frac{\alpha}{2}\right)=0$;

$$
\begin{array}{ll}
\lambda_{b}=2 b \frac{\pi}{\alpha}, & \text { with } b=1,2, \ldots, \\
\lambda_{b}^{*}=(2 b-1) \frac{\pi}{\alpha}, & \text { with } b=1,2, \ldots .
\end{array}
$$


Singularities can be present if $\alpha>\pi$ for the anti-symmetric set.

- Dirichlet-Neumann boundary conditions: $p\left(r,-\frac{\alpha}{2}\right)=v_{\theta}\left(r, \frac{\alpha}{2}\right)=0$;

$$
\lambda_{b}=\lambda_{b}^{*}=\pi /(2 \alpha)+2(b-1) \frac{\pi}{\alpha}, \quad \text { with } b=1,2, \ldots \text { and } A_{\lambda_{b}}=A_{\lambda_{b}^{*}} .
$$

Singularities can be present if $\alpha>\pi / 2$.

- Neumann-Dirichlet boundary conditions: $v_{\theta}\left(r,-\frac{\alpha}{2}\right)=p\left(r, \frac{\alpha}{2}\right)=0$;

$$
\lambda_{b}=\lambda_{b}^{*}=\pi /(2 \alpha)+2(b-1) \frac{\pi}{\alpha}, \quad \text { with } b=1,2, \ldots \text { and } A_{\lambda_{b}}=-A_{\lambda_{b}^{*}} .
$$

Singularities can be present if $\alpha>\pi / 2$.

For non-homogeneous boundary conditions, generally no closed dynamic solutions can be found due to the $r$-dependence in the Bessel functions.

\subsection{Static solutions of the infinite wedge}

To be able to predict whether singularities are present for non-homogeneous boundary conditions, the dynamic $(k>0)$ solutions of the infinite wedge are compared with the static $(k=0)$ solutions, similarly as is proposed in [37] for plate bending problems. Instead of the Helmholtz equation (1), the Laplace equation $\nabla^{2} p(\boldsymbol{r})=0$ is thus solved. The analytical solutions of the Laplace equation in an infinite wedge considering all combinations of imposed homogeneous Neumann or Dirichlet boundary conditions are given by Li and Lu [3] and are shown in Table 1. The results are obtained in a shifted coordinate system $(0 \leq \theta \leq \alpha)$. As was already verified by Millar [48] in 1971, the solutions of the Laplace and Helmholtz equations that satisfy the same Dirichlet and Neumann conditions on the same boundary, possess the same convex hull of singularities. The powers of $r$ which occur in the elementary solutions of the Laplace equation have to be replaced by Bessel functions of the first kind with argument $k r$ to obtain the elementary solutions of the Helmholtz equation for the similar problem. As can easily be verified, by comparing the analytical solutions for the infinite wedge for the Laplace equation in Table 1 with the ones for the Helmholtz equations, applying the same boundary conditions (see [16] for the same coordinate system), indeed exactly the same singular exponents $\lambda$ are found. Hence, the following relation exists between the static and the dynamic eigenvalues:

$$
\lambda_{\text {dynamic }}=\lambda_{\text {static }} .
$$

As a consequence, the equivalent static problem can be used to predict the existence of singularities and the accompanying eigenvalues for the dynamic Helmholtz problem. Even in the case of non-homogeneous boundary conditions, for which no dynamic solutions of the Helmholtz equation can be found, it is possible to define solutions of the Laplace equation. This way, it can be verified if non-homogeneous boundary conditions may lead to additional singularities. $\mathrm{Li}$ and $\mathrm{Lu}[3]$ also give particular solutions of the Laplace equation that need to be added to the homogeneous particular solutions of Table 1 in the case when the imposed boundary conditions are constant but different from zero. 


\begin{tabular}{lll}
\hline Boundary conditions & Homogeneous particular solution & Exponent \\
\hline Neumann-Neumann & $p(r, \theta)=\sum_{b=0}^{\infty} a_{b} r^{\lambda_{b} \cos \left(\lambda_{b} \theta\right)}$ & $\lambda_{b}=\frac{b \pi}{\alpha}$ \\
Dirichlet-Dirichlet & $p(r, \theta)=\sum_{b=0}^{\infty} a_{b} r^{\lambda} \sin \left(\lambda_{b} \theta\right)$ & $\lambda_{b}=\frac{b \pi}{\alpha}$ \\
Neumann-Dirichlet & $p(r, \theta)=\sum_{b=0}^{\infty} a_{b} r^{\lambda_{b}} \cos \left(\lambda_{b} \theta\right)$ & $\lambda_{b}=\frac{\pi}{\alpha}(b+1 / 2)$ \\
Dirichlet-Neumann & $p(r, \theta)=\sum_{b=0}^{\infty} a_{b} r^{\lambda_{b}} \sin \left(\lambda_{b} \theta\right)$ & $\lambda_{b}=\frac{\pi}{\alpha}(b+1 / 2)$ \\
\hline
\end{tabular}

Table 1: Particular solutions of the Laplace equation for an infinite wedge $(0 \leq \theta \leq \alpha)$ for different combinations of homogeneous boundary conditions [3].

\begin{tabular}{llll}
\hline Boundary conditions & $\begin{array}{l}\text { Imposed values } \\
\text { leading to singularity }\end{array}$ & Angle & Singular particular solution \\
\hline Neumann-Neumann* & $(-1)^{l} B \neq A$ & $\alpha=\pi, 2 \pi$ & $\frac{(-1)^{l} B-A}{\alpha} r(\ln r \cos \theta-\theta \sin \theta)$ \\
Dirichlet-Dirichlet & $A \neq B$ & all angles & $A+\frac{(B-A) \theta}{\alpha}$ \\
Neumann-Dirichlet & $A \neq 0$ & $\alpha=\pi / 2,3 \pi / 2$ & $-\frac{A r}{\alpha}(\ln r \cos \theta-\theta \sin \theta)$ \\
Dirichlet-Neumann* & $B \neq 0$ & $\alpha=\pi / 2,3 \pi / 2$ & $\frac{(-1)^{l+1} B r}{\alpha}(\ln r \sin \theta+\theta \cos \theta)$ \\
\hline
\end{tabular}

Table 2: Existence of logarithmic singularities of the Laplace equation [3], ${ }^{*} l=0$ for the first angle, $l=1$ for the second angle.

For a wide range of internal angles, this leads to the addition of some particular terms that do not possess an additional singularity in the corner point. Concerning the dynamic equivalent Helmholtz equation, for these cases also no additional singularities are expected. However, for some combinations of specific angles and boundary conditions additional singularities can be found. These cases are given in Table 2 [3], listing the imposed boundary conditions, the angles for which an additional singularity is found and the singular term of the particular solution. The imposed value on the first boundary $(\theta=0)$ is equal to $A$ and $B$ is the imposed value on the second boundary $(\theta=\alpha)$.

As shown in Table 2, for Dirichlet-Dirichlet boundary conditions additional singularities are present if two different pressures are prescribed on both sides of the corner point. This is a non physical situation, since the pressure is then discontinuous, and is therefore not further considered. For the other combinations of boundary conditions in Table 2, for particular angles, logarithmic fields are present. Logarithmic singularities were also discussed by Sinclair [49] and can exist when

$$
\mathcal{D}=0 \text { and } \frac{\partial \mathcal{D}}{\partial \lambda}=0
$$

with $\mathcal{D}$ the determinant of the static coefficient matrix $\mathbf{A}$, as is discussed for the Helmholtz equation in Appendix A. In this case, auxiliary fields come into play, as is also the case for the Laplace equation as demonstrated in [3] and the solutions of Table 2 are found. However, defining analogously dynamic auxiliary fields is impossible since no analytical solution exists for a derivative of a Bessel function with respect to its order. It is therefore impossible to make the static and dynamic solutions asymptotically equivalent only by adequately choosing the dynamic eigenvalues. As a result, currently no dynamic solution of the Helmholtz equation can be defined, that accurately represents the singularities in the velocity field when logarithmic singularities are present for the Laplace equation. Nevertheless, singularities of this type only occur in a rare number of specific configurations as given in Table 2 . The effect of the presence of a logarithmic singularity on the convergence behaviour of the WBM will be studied in one of the 
numerical examples in section 5 .

\subsection{Robin boundary conditions}

For Robin boundary conditions it is impossible, both for the Helmholtz equation and for the Laplace equation, to find closed form analytical solutions for an infinite wedge. Nevertheless, also here we can apply the reasoning that the solution can be represented by a series fulfilling the homogeneous boundary conditions and some particular terms taking the non-homogeneous boundary conditions into account. The latter may conceivably lead again to logarithmic singularities, but these can anyway not be accounted for with dynamic particular solutions. If we consider the 'homogeneous' case and apply $Z_{n}=0$, which is the ratio of $p(\boldsymbol{r})$ and $v_{\theta}(\boldsymbol{r})$ on the boundary, it can be seen as imposing Dirichlet or Neumann boundary conditions. The singularity is then expected to have the same order as for the Dirichlet-Dirichlet or Neumann-Neumann case. A validation case within the verification section examines if adding similar enrichment functions for the Robin-Robin combination as for the Neumann-Neumann case improves the convergence of the WBM.

\subsection{Application within the WBM}

An important remark when applying special purpose enrichment functions, from here onwards called corner functions, within the WBM, is that they do not form a Trefftz-complete set. Consequently, by only using corner functions in the wave function set, the convergence towards the exact solution of the problem is not ensured. As such, they can only be used in combination with the conventional set of wave functions. Also, since the corner functions do not fulfil the Sommerfeld radiation condition (6), singular corners should be included within the bounded subdomains inside the truncation circle $\Gamma_{t}$. The corner functions will cope with the singular behaviour resulting from the homogeneous boundary conditions, whereas the regular wave functions and the particular solutions will assure that the boundary conditions and point sources are correctly taken into account. For each corner $c_{i}$ of the problem domain where a singularity exists, a set of corner functions will be added to the field variable expansion of the bounded subdomain(s) to which the corner belongs. It can be shown by calculating the Wronskian [50] that the proposed corner functions are linearly independent from the regular wave functions and consequently do not lead to singular WB matrices. Figure 4 explains the principle of addition of corner functions to wave function sets. The shown problem domain is divided into two convex subdomains $\Omega^{(1)}$ and $\Omega^{(2)}$. Suppose the domain contains two singular corners $c_{1}$ and $c_{2}$. Each corner function is defined in a local coordinate system attached to the corner point (the corner $c_{\bullet}$ is the origin, $\theta$ is related with the bisecting line $(\cdots)$ of the corner). Contrary to wave functions, corner functions are not necessarily restricted to one subdomain. For example corner $c_{2}$ is associated with one subdomain, but corner $c_{1}$ belongs to both subdomains $\Omega^{(1)}$ and $\Omega^{(2)}$. Therefore, the corner functions belonging to this corner are added to both wave functions expansions $\hat{p}^{(1)}(\boldsymbol{r})$ and $\hat{p}^{(2)}(\boldsymbol{r})$. These corner functions are continuous over the interface $\Gamma_{I}^{(\alpha, \beta)}$ and are excluded from the residuals of that interface. Just like ordinary wave functions, each corner function is multiplied with an unknown contribution factor. Since the purpose of the corner functions is to accurately represent the singularity, only the eigenfunctions 


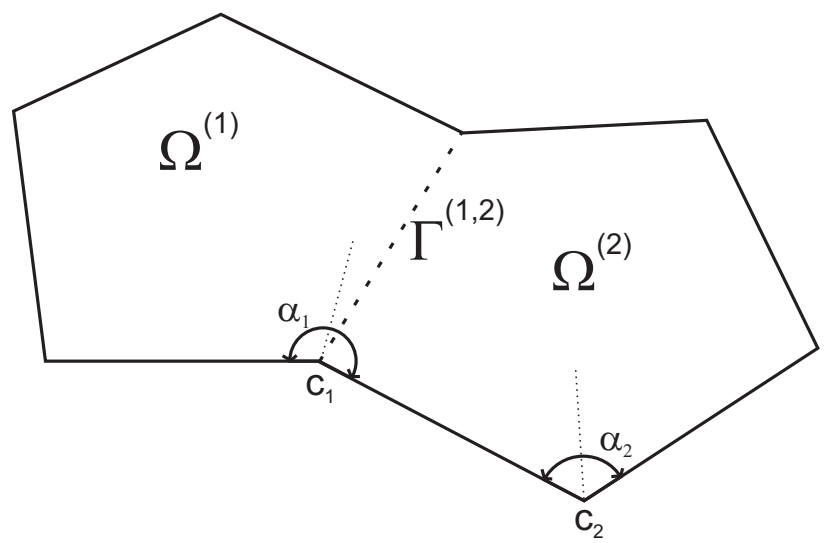

Figure 4: Application of corner functions in the WBM.

with eigenvalues $0<\lambda<1$ are included in the wave function set. The pressure field approximation (8) is extended with the corner functions contributions $\Psi_{c_{i}}$ for each singular corner $c_{i}$ in subdomain $\Omega^{(\alpha)}$ :

$$
p^{(\alpha)}(\boldsymbol{r}) \simeq \hat{p}^{(\alpha)}(\boldsymbol{r})=\sum_{w=1}^{n_{w}^{(\alpha)}} p_{w}^{(\alpha)} \Phi_{w}^{(\alpha)}(\boldsymbol{r})+\hat{p}_{q}(\boldsymbol{r})+\sum_{c_{i}^{(\alpha)}} p_{c_{i}}^{C F} \Psi_{c_{i}}^{(\alpha)}\left(r_{c_{i}}, \theta_{c_{i}}\right),
$$

with $p_{c_{i}}^{C F}$ the contribution factor of corner function $\Psi_{c_{i}}^{(\alpha)}$. The same corner functions are also applied as weighting functions. This way, one obtains a sufficient number of equations to also determine $p_{c_{i}}^{C F}$.

\section{Verification examples}

This section demonstrates the beneficial efficiency of adding corner functions to the WBM in the case when acoustic singularities are present. In all examples the fluid properties for standard air $\left(\rho=1.225 \mathrm{~kg} / \mathrm{m}^{3}, c=340 \mathrm{~m} / \mathrm{s}\right)$ are used. Firstly, the well-known Motz's problem is studied. The efficiency of the adapted WBM (WBM CF) is compared with the WBM without special purpose functions, both concerning accuracy and calculation time. Next, an L-shaped domain is considered. For this geometry various problem settings are assessed. Lastly, an unbounded verification case shows that the same principles can be applied in unbounded acoustic cases. In this example the scattering of a star-shape domain with four singular corners is considered. All WB routines are implemented in Matlab R2010a and the models are solved using Matlab's backslash, i.e. by Gaussian elimination. To obtain independent references for each of the models, very fine FE or BE models are used. The FE predictions of the bounded problems are obtained using the acoustic module of Comsol 3.5a. In this case, quadratic elements are used. Also adaptive refinements based on an $L^{2}$-norm of the error in the domain are performed to speed up convergence. The FE models are solved using a direct UMFPACK solver. For the unbounded case, a reference for the WBM is obtained with indirect BE models, calculated with LMS Sysnoise 5.6. In this case an in-core Blocked Cholesky solver is applied. The calculation times include both the construction and the solution times. All calculations are performed on a Linuxbased 2.66 GHz Intel Xeon system with 32GB RAM. 


\subsection{Acoustic Motz's problem}

The first example considers the well-known Motz's problem [51] for acoustics. The problem description is given in Figure 5. The rectangular cavity has dimensions $2 \mathrm{mx} 1 \mathrm{~m}$. The solid black lines (-) indicate a sound hard wall $\left(\bar{v}_{n}=0 \mathrm{~m} / \mathrm{s}\right)$, the dashed boundary (- $)$ is a sound soft boundary $(\bar{p}=0 \mathrm{~Pa})$ and on the dashed boundary with markers (-o-) an acoustic pressure of $1 \mathrm{~Pa}$ is imposed. The figure also indicates the subdivision into two WB subdomains.

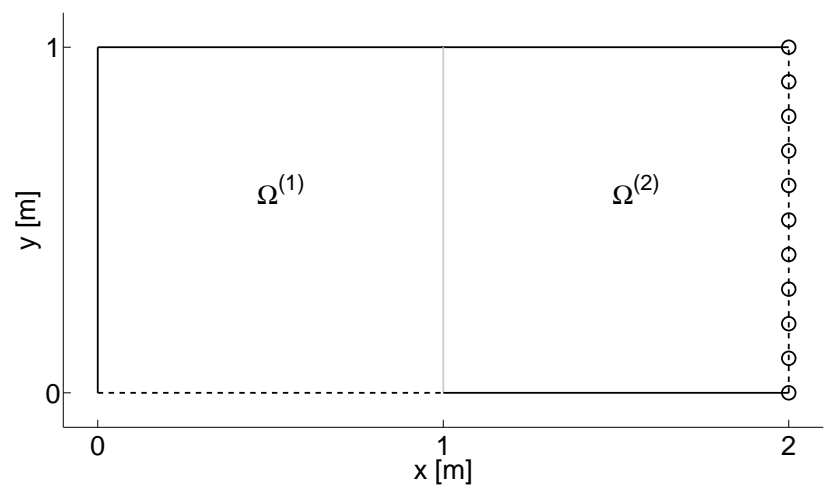

Figure 5: Motz's problem description.

A singularity exists in the point $(1,0)$ where the boundary conditions change from Neumann to Dirichlet. Using expression (34) the eigenvalues $\lambda^{*}=\lambda=1 / 2$ lead to a singularity in the velocity field and the corner function

$$
\Psi_{c_{1}}^{C F}=(\sin (1 / 2 \theta)-\cos (1 / 2 \theta)) J_{1 / 2}(k r)
$$

is added to the wave function expansions of both subdomains. The order of the singularity in the velocity field is $-1 / 2$. Figure 6 shows the absolute value of the $v_{y}$-field calculated with the WBM CF at $450 \mathrm{~Hz}$. A steep gradient can be seen in the near vicinity of the singular point.

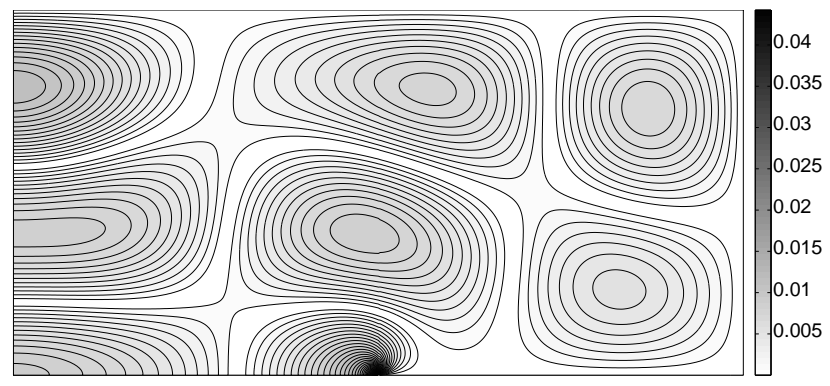

Figure 6: Contour plot of the absolute value of $v_{y}(x, y)[\mathrm{m} / \mathrm{s}]$ at $450 \mathrm{~Hz}$ calculated with the WBM including a corner function.

To validate the applicability of the method for a wider frequency range, frequency response functions (FRFs) of the absolute value of the acoustic pressure in an arbitrary point $(0.5,0.25)$ are shown in Figure 7 . For the WBM $\mathrm{CF}$ results a fixed truncation $N=4$ is used. The FE reference is obtained calculating 10 adaptive refinements per 
frequency, resulting in approximately 650.000 degrees of freedom (DOFs). The curves coincide, indicating a good agreement between the results obtained with both methods.

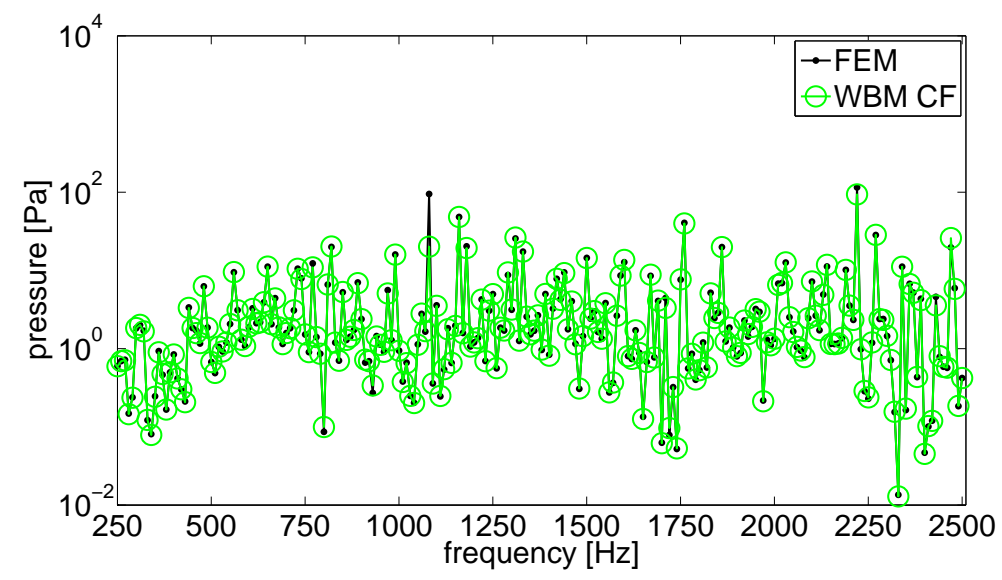

Figure 7: Frequency response function of the pressure in point $(0.5,0.25)$ calculated with both the WBM CF and the FEM.

Figure 8 shows the relative error between the results obtained with the WBM CF and the WBM $(N=4)$ respectively and the FEM in the same point $(0.5,0.25)$ over the same frequency range. The error is also averaged over $1 / 3$ octave bands to show the trends more clearly. As compared to the WBM, the WBM CF wave function set only contains one additional function. The results clearly improve.

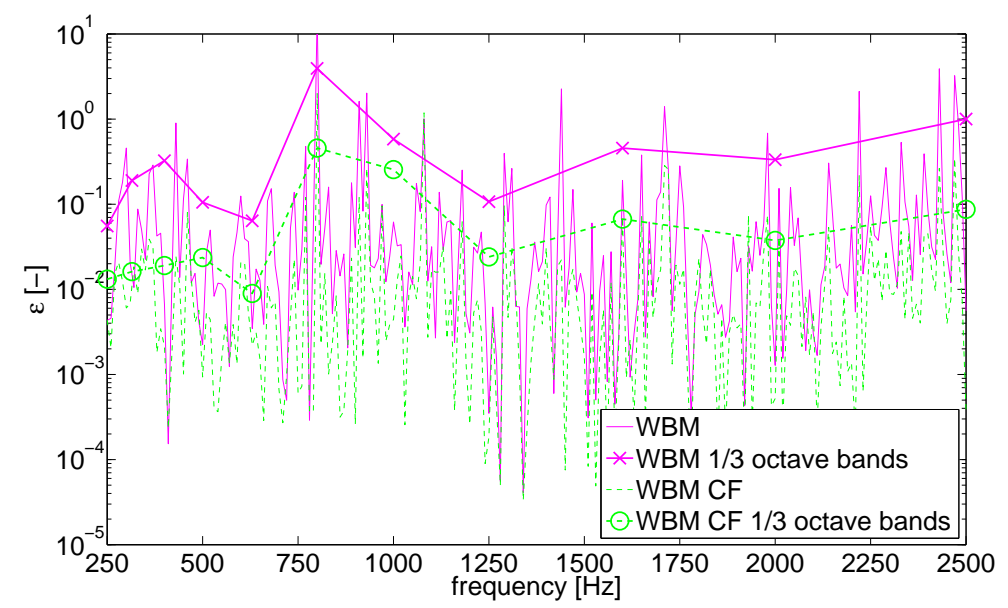

Figure 8: Error FRFs of the pressure in point $(0.5,0.25)$ calculated with both the WBM and the WBM CF and the FEM as a reference.

To compare the convergence rate of the different methods, the averaged relative prediction error $\langle\epsilon>$ of the obtained pressure field is calculated as a function of CPU time. The prediction error in a certain response point is calculated as the absolute value of the relative error between the calculated value of the pressure in the response point and the reference value in that point. This prediction error is averaged over $n$ response points which are equally 


\begin{tabular}{|c|c|c|c|c|c|c|c|c|}
\hline \multicolumn{9}{|c|}{ FEM quadratic adaptive } \\
\hline \multirow{4}{*}{$450 \mathrm{~Hz}$} & $\sharp$ refs & 0 & 1 & 2 & 3 & 4 & 5 & 6 \\
\hline & $\sharp \mathrm{DOFs}$ & 694 & 1.433 & 2.723 & 5.280 & 11.078 & 22.757 & 45.770 \\
\hline & $\sharp$ refs & 7 & 8 & 9 & 10 & 11 & 12 & \\
\hline & $\sharp \mathrm{DOFs}$ & 92.545 & 183.464 & 368.585 & 727.608 & 1.451 .701 & 2.869 .259 & \\
\hline \multirow{4}{*}{$1035 \mathrm{~Hz}$} & $\sharp$ refs & 0 & 1 & 2 & 3 & 4 & 5 & 6 \\
\hline & $\sharp \mathrm{DOFs}$ & 694 & 1.386 & 2.687 & 5.299 & 10.358 & 21.101 & 40.992 \\
\hline & $\sharp$ refs & 7 & 8 & 9 & 10 & 11 & 12 & \\
\hline & $\sharp \mathrm{DOFs}$ & 83.091 & 16.2050 & 328.708 & 639.096 & 1.294 .073 & 2.521 .077 & \\
\hline \multicolumn{9}{|c|}{ FEM quadratic } \\
\hline $450 \mathrm{~Hz} \&$ & $\sharp$ refs & 0 & 1 & 2 & 3 & 4 & 5 & 6 \\
\hline $1035 \mathrm{~Hz}$ & $\sharp D O F s$ & 2.679 & 10.525 & 41.721 & 166.129 & 663.009 & 2.649 .025 & 10.590 .081 \\
\hline
\end{tabular}

Table 3: FE model data for Motz's problem.

distributed over the acoustic domain:

$$
<\epsilon>=\frac{1}{n} \sum_{j=1}^{n} \epsilon_{j} \quad \text { with } \quad \epsilon_{j}=\left|\frac{p\left(\boldsymbol{r}_{j}\right)-p^{r e f}\left(\boldsymbol{r}_{j}\right)}{p^{r e f}\left(\boldsymbol{r}_{j}\right)}\right| .
$$

In this case, $n=21$ equidistantly distributed postprocessing points are used. Convergence curves are calculated with the WBM and WBM CF as well as with globally and adaptively refined quadratic FE models. The details of the FE models are given in Table 3. Between two subsequent quadratic adaptively refined FE models, one adaptive refinement is performed, based on the $L^{2}$-norm of the error in the domain. The number of subsequent refinements (\#refs) is also given. Figure 9 shows the basis FE mesh and the mesh after three adaptive refinements at $450 \mathrm{~Hz}$. The mesh is clearly refined around the singular corner where the gradients in the field are highest. The quadratic adaptive model that applies 12 subsequent adaptive refinements is taken as a reference. For the quadratic FE models, the meshes are frequency independent. For each refinement, each triangular element was divided into four triangular elements.

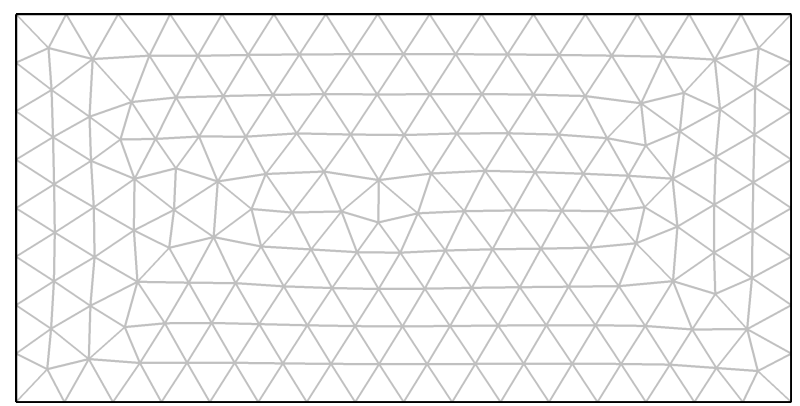

(a) basis FE mesh, 0 adaptive refinements

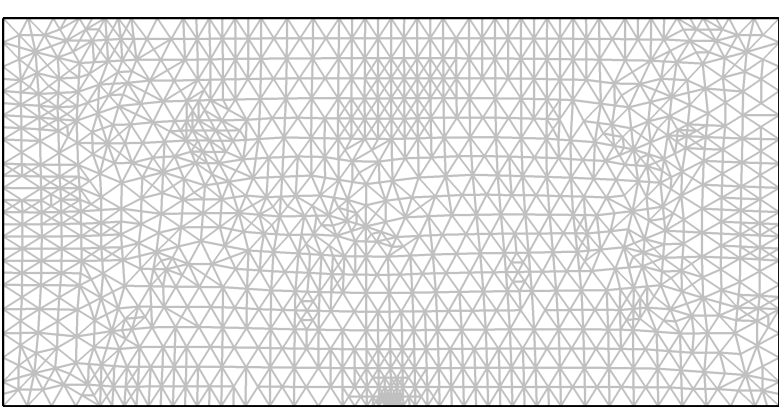

(b) FE mesh after three adaptive refinements

Figure 9: FEM quadratic adaptive meshes, (a) basis mesh, (b) mesh after three adaptive refinements at $450 \mathrm{~Hz}$.

To calculate the WB convergence curves, the number of wave functions is continuously increased according to the frequency dependent truncation rule (14). For the first model a truncation factor $N=1$ and for the finest model a 
truncation factor $N=100$ is applied. In between these extreme values a variable spacing is applied, such that more samples are taken for lower truncation factors and less for higher truncation factors. The convergence curves of the WBM for this validation case are obtained applying the following values of $N$ : [1 234567810141820253040 5060708090 100]. Since the bounding boxes are square the r- and the s-set as defined in equation (9) contain an equal number of wave functions.

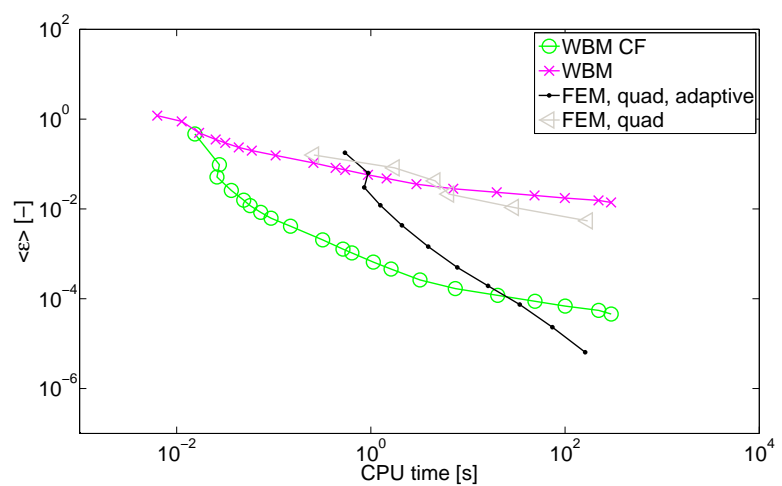

(a) $450 \mathrm{~Hz}$

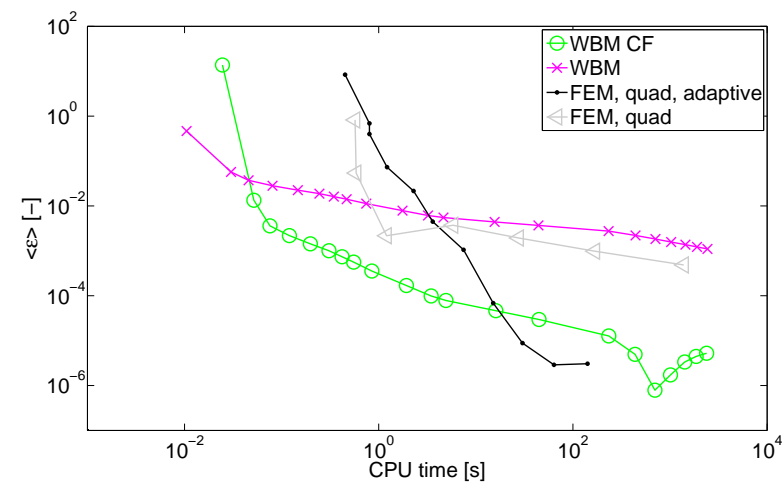

(b) $1035 \mathrm{~Hz}$

Figure 10: Convergence curves at two frequencies for the WBM, the WBM CF, quadratic FEM and quadratic adaptive FEM.

Figure 10 compares the convergence curves of the WBM, the WBM CF, and the FE models at $450 \mathrm{~Hz}$ and $1035 \mathrm{~Hz}$ respectively. The beneficial effect of adding corner functions to the wave function expansion is clearly illustrated. While the WBM converges similarly as the quadratic FEM, the convergence rate of the WBM CF is much improved at a very small extra cost, shown as an offset in CPU time. The figure also illustrates the problems the FEM encounters; a sufficiently refined mesh near the corner has to be applied to obtain an accurate reference.

\subsection{L-shape problem}

As a second example, an L-shaped problem domain as shown in Figure 11 is considered. It has a reentrant corner at $(0.5,0.5)$. The problem domain is divided into three equal square WB subdomains as indicated in the figure. Depending on the boundary conditions imposed on the chain-dotted boundaries, $\Gamma_{1}$ and $\Gamma_{2}$, different types of singularities can be expected. The solid boundaries are acoustically rigid for all calculation cases. Depending on the considered case, on the dashed boundary $\Gamma_{3}$ a normal velocity of $0 \mathrm{~m} / \mathrm{s}$ or $1 \mathrm{~m} / \mathrm{s}$ is imposed. The figure also indicates the subdivision into three convex WB subdomains.

Various studies are performed on this geometry:

- Firstly, the effect of different types of excitation is examined, i.e. excitation by an acoustic point source at point $\mathrm{Q}$ or an imposed normal acoustic velocity of $1 \mathrm{~m} / \mathrm{s}$ at the dashed boundary $\Gamma_{3}$.

- It is investigated if adding more analytical solutions of the infinite wedge case leads to a more efficient solution or not as compared to adding just the corner functions that incorporate the singularity. 


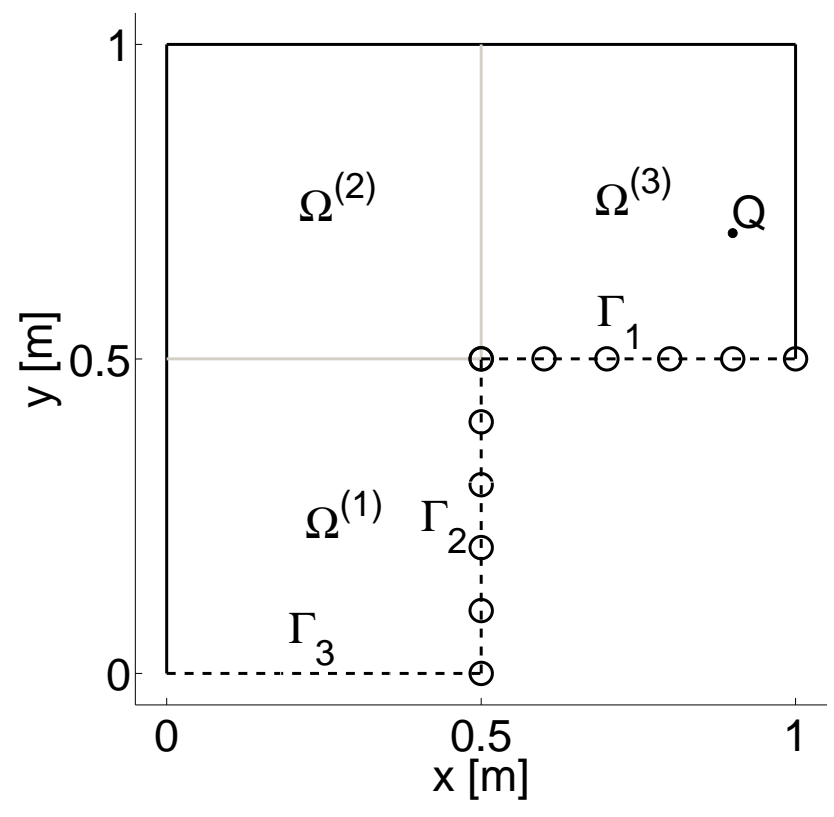

Figure 11: L-shape problem description.

- Next, different combinations of boundary conditions are applied at $\Gamma_{1}$ and $\Gamma_{2}$ and the effect on the convergence is studied. Also the case of Robin-Robin boundary conditions is tackled.

- The last study includes a logarithmic singularity.

Convergence curves are calculated for the four different cases at two arbitrary frequencies, $420 \mathrm{~Hz}$ and $800 \mathrm{~Hz}$, to verify the improvement between the WBM and the WBM CF. Also for these cases, for the coarsest model and the finest WB model truncation factors $N=1$ and $N=100$ are applied respectively, see equation (14). As for Motz's problem case, to

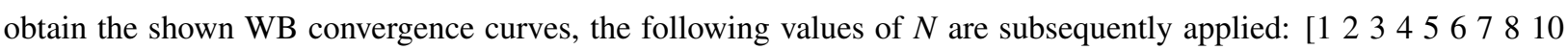
1418202530405060708090 100]. Since the dimensions of the bounding box of each of the three subdomains are equal, the same number of wave functions is applied in each subdomain and the $r$ - and the s- set contain the same number of wave functions since the bounding boxes are square.

Very accurate adaptively refined FE models containing quadratic meshes are taken as a reference. Further refinements were impossible due to memory restrictions of the available hardware. Table 4 contains the details of the FE reference models of the different cases. For all convergence curves of the L-shape cases, the shown relative error is calculated using equation (38), applying $n=63$ distributed response points.

\subsubsection{Homogeneous versus non-homogeneous Helmholtz equation}

The first case studies the L-shape with Neumann boundary conditions on $\Gamma_{1}$ and $\Gamma_{2}$ for the homogeneous and a non-homogeneous Helmholtz equation and verifies if a similar improvement can be obtained by adding a corner function. 


\begin{tabular}{lll}
\hline case & $420 \mathrm{~Hz}$ & $800 \mathrm{~Hz}$ \\
\hline NN H & $7,907,006$ & $5,143,904$ \\
NN NH & $4,907,304$ & $7,016,530$ \\
DD NH & $8,867,890$ & $6,057,028$ \\
DN NH & $9,4181,99$ & $9,019,907$ \\
RR NH & $7,405,112$ & $6,006,976$ \\
DN H & $9,359,522$ & $6,209,001$ \\
DN H log & $7,567,107$ & $5,606,992$ \\
\hline
\end{tabular}

Table 4: Number of DOFs for the FE reference models for the L-shape problem cases (H=homogeneous Helmholtz equation, NH=nonhomogeneous Helmholtz equation).

When applying the homogeneous Helmholtz equation, the domain is excited with a normal acoustic velocity of $1 \mathrm{~m} / \mathrm{s}$ on boundary $\Gamma_{3}$ and the other edges are acoustically rigid. For the non-homogeneous case, an acoustic point source with amplitude $q=1 \mathrm{~Pa}$ at point $\mathrm{Q}(0.9,0.7)$ excites the problem domain and all edges are considered sound hard. For both cases, the analytical solutions of the infinite wedge with homogeneous Neumann-Neumann boundary conditions, equation (32) have to be applied. The only eigenvalue leading to a singularity in the velocity field is $\lambda_{1}^{*}=2 / 3$. Consequently,

$$
\Psi_{c_{1}}^{C F}=\sin (2 / 3 \theta) J_{2 / 3}(k r)
$$

is added to the wave function set of the three subdomains for the WBM CF for both the homogeneous and the nonhomogeneous problem.

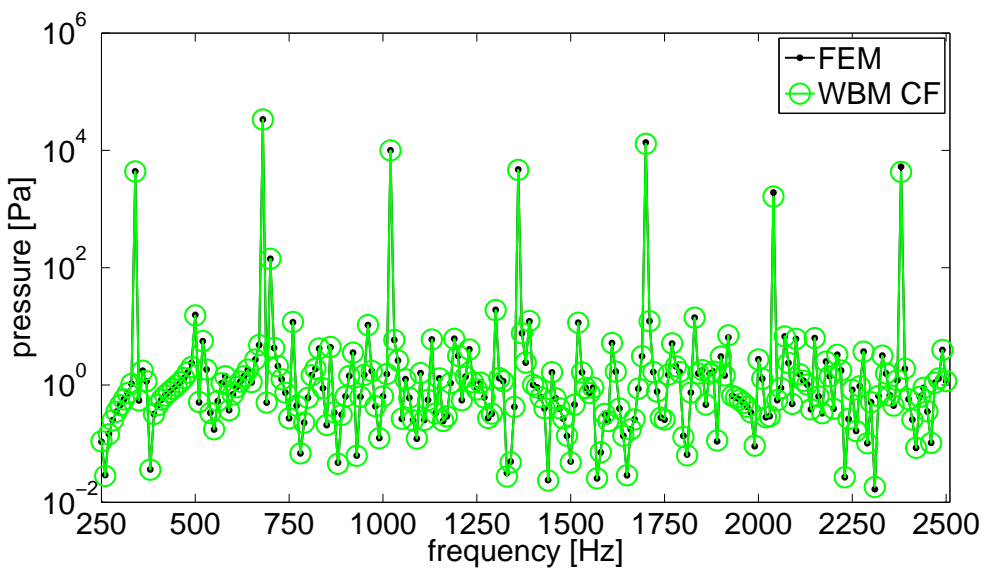

Figure 12: Frequency response function of the pressure in point $(0.3,0.2)$ calculated with both the WBM CF and the FEM.

Figure 12 shows the FRF of the acoustic pressure in a response point with coordinates $(0.3,0.2)$, obtained with the WBM CF and the FEM for the homogeneous case. For the WB model, a truncation factor $N=4$ was applied. For the FEM, 9 adaptive refinements were performed at each frequency line to obtain accurate reference results, leading to approximately 500.000 DOFs per frequency line. No visual difference can be seen between both curves indicating that a good accuracy is obtained. Figure 13 shows the relative error between the results obtained with the WBM CF 


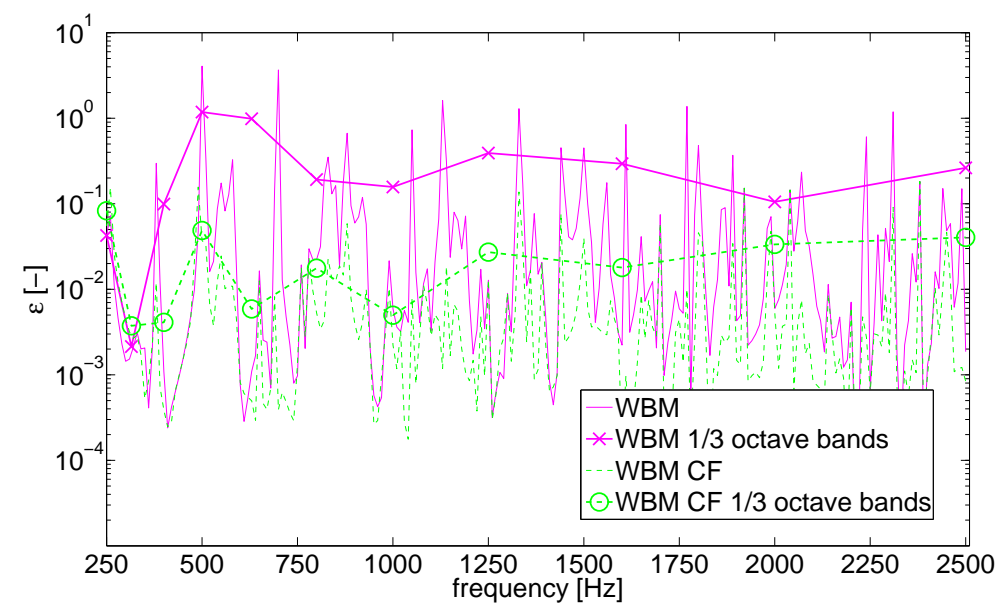

Figure 13: Error FRFs of the pressure in point $(0.3,0.2)$ of the L-shape calculated with both the WBM and the WBM CF and the FEM as a reference.

and the WBM $(N=4)$ respectively and the FEM in the same point $(0.3,0.2)$ over the same frequency range. The error is also shown averaged over 1/3 octave bands. Also here, similarly as for Motz's example, the addition of one corner function clearly improves the results.

Figure 14 shows the convergence curves of the acoustic pressure, obtained with the WBM and the WBM CF for both the homogeneous Helmholtz problem $(\mathrm{H})$ and the non-homogeneous Helmholtz problem (NH) at two distinct frequencies. The FE models indicated by NN H and NN NH of Table 4 are used as references. The convergence behaviour for both cases is similar. Due to the evaluation of the particular solution to account for the point source (16) on the boundaries which is more expensive than the evaluation of the wave functions, the curves shift a bit to the right. Also, since it is more difficult to account for the field of a point source, the curves shift up a little, but the convergence rate is similar. The addition of the corner function which takes into account the singularity, leads to a significant increase in accuracy at a small extra cost. For the homogeneous case as well as for the non-homogeneous case, a similar improvement is obtained at both frequencies, as was expected.

\subsubsection{Effect of adding more corner functions}

As explained in section 4.1, a series of exact solutions of the infinite wedge problem with homogeneous boundary conditions is found. Within the WBM, only the corner functions leading to infinite values of the velocity field generally are added to the wave function expansion [37]. This case studies the effect on the convergence of the WBM when more analytical solutions of the infinite wedge are added to the wave function set. As such, it can be evaluated whether the addition of the corner functions leading to a singular velocity field is optimal, or if the convergence rate can be further improved by adding even more corner functions.

The non-homogeneous L-shape problem with Neumann-Neumann boundary conditions is again studied. The convergence of five different WB models are compared; the first one applies no corner function, the second one applies only the first analytical solution of the infinite wedge problem, the third one applies two analytical solutions 


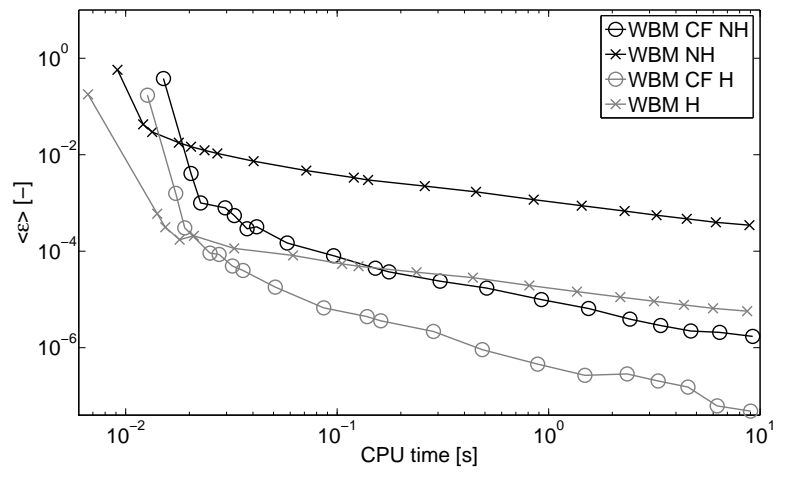

(a) $420 \mathrm{~Hz}$

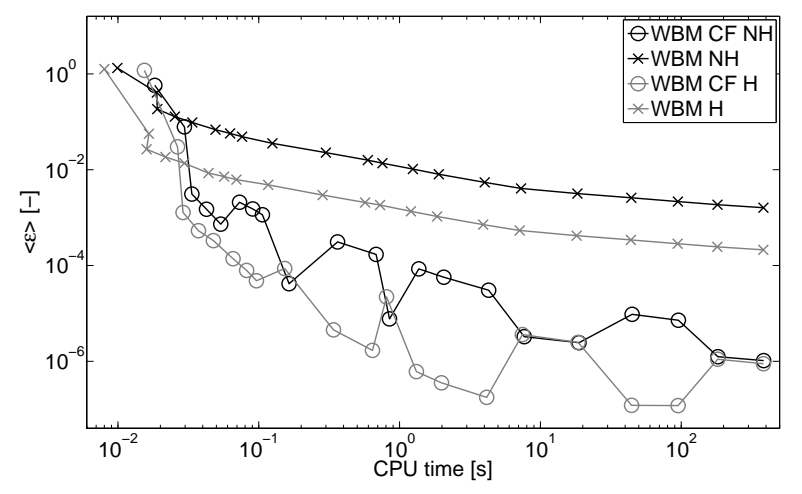

(b) $800 \mathrm{~Hz}$

Figure 14: Convergence curves of the WBM and the WBM CF for the homogeneous $(\mathrm{H})$ and non-homogeneous Helmholtz equation at 420 and $800 \mathrm{~Hz}$.

\begin{tabular}{lll}
\hline Nr. & eigenvalue & Corner function \\
\hline 1 & $\lambda_{1}^{*}=2 / 3$ & $\sin (2 / 3 \theta) J_{2 / 3}(k r)$ \\
2 & $\lambda_{1}=4 / 3$ & $\cos (4 / 3 \theta) J_{4 / 3}(k r)$ \\
3 & $\lambda_{2}^{*}=2$ & $\sin (2 \theta) J_{2}(k r)$ \\
4 & $\lambda_{2}=8 / 3$ & $\cos (8 / 3 \theta) J_{8 / 3}(k r)$ \\
\hline
\end{tabular}

Table 5: First four analytical solutions of the infinite wedge applying homogeneous Neumann-Neumann boundary conditions.

and so forth. The first four corner functions, based on equations (31)-(32) are given in Table 5 and only the first one leads to a singularity in the velocity field. The FE model indicated by NN H in Table 4 is again used as a reference.

Figure 15 shows the convergence of the five different WB models. The conventional set of wave functions is extended in a similar way as in the previous example. Each addition of an extra corner function leads to an increase of CPU time. The differences in obtained accuracies are negligible. This study confirms that the addition of only the special purpose functions that capture the singular behaviour is sufficient. The convergence rate is not noticeably changed by adding more corner functions. Only a larger calculation time is required.

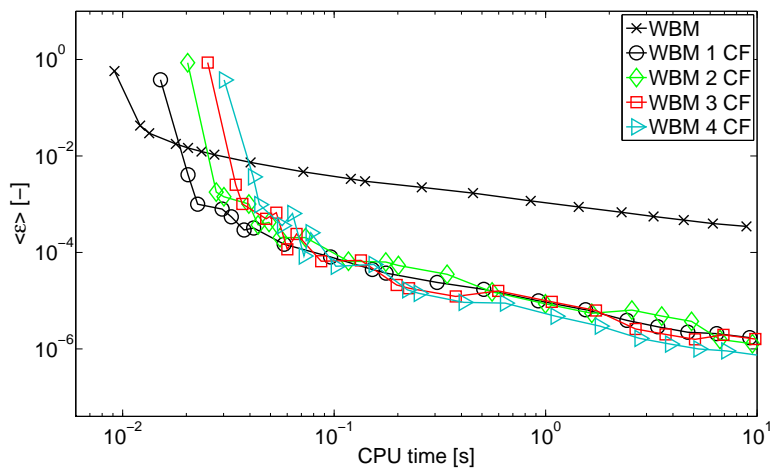

(a) $420 \mathrm{~Hz}$

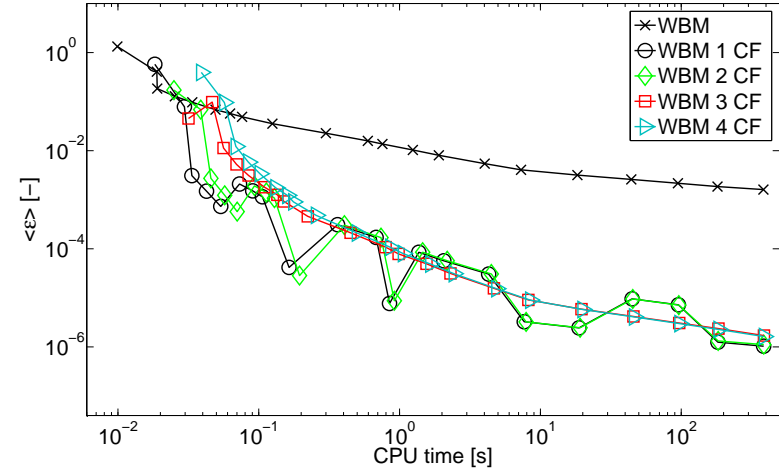

(b) $800 \mathrm{~Hz}$

Figure 15: Convergence curves of the WBM with an increasing number of wave functions at different frequencies. 


\begin{tabular}{llll}
\hline Boundary Conditions & eigenvalue & Corner function & notation \\
\hline Neumann-Neumann & $\lambda^{*}=2 / 3$ & $\sin (2 / 3 \theta) J_{2 / 3}(k r)$ & $\mathrm{NN}$ \\
Dirichlet-Neumann & $\lambda=\lambda^{*}=1 / 3$ & $(\sin (1 / 3 \theta)+\cos (1 / 3 \theta)) J_{1 / 3}(k r)$ & $\mathrm{DN}$ \\
Dirichlet-Dirichlet & $\lambda=2 / 3$ & $\cos (2 / 3 \theta) J_{2 / 3}(k r)$ & $\mathrm{DD}$ \\
Robin-Robin & $\lambda^{*}=2 / 3$ & $\sin (2 / 3 \theta) J_{2 / 3}(k r)$ & $\mathrm{RR}$ \\
\hline
\end{tabular}

Table 6: Different combinations of boundary conditions imposed on $\Gamma_{1}$ and $\Gamma_{2}$ for four different cases and the applied corner functions.

\subsubsection{Different boundary conditions}

Next, different boundary conditions are imposed on $\Gamma_{1}$ and $\Gamma_{2}$ of the L-shape in Figure 11 to evaluate if the beneficial effect of the additional corner function is similar. Boundary $\Gamma_{3}$ is a sound hard wall and a point source with $q=1 \mathrm{~Pa}$ excites the problem domain at point Q. Four different combinations of boundary conditions are compared and are listed in Table 6. For the three first cases, homogeneous boundary conditions are imposed and the solutions of the corresponding infinite wedge case leading to a singularity are added as corner functions. For the Robin-Robin case on both boundaries a normal impedance $\bar{Z}_{n}$ of $441+j 1241 \mathrm{~Pa} \cdot \mathrm{s} / \mathrm{m}$ is imposed. As described in section 4.4 , in this case no analytical solution is known, fulfilling the Helmholz equation as well as the imposed boundary conditions. As proposed in section 4.4, the same corner function as for the Neumann-Neumann case is added to the wave function expansion. The singularity for the Dirichlet-Neumann case is the most severe of all the studied cases in this subsection. Figure 16 shows the convergence of the four cases at 420 and $800 \mathrm{~Hz}$ respectively. The details of the FE reference models are given in Table 4 and the models NN NH, DD NH, DN NH and RR NH are used here. At 420 Hz, the convergence behaviour of the WB models without CF is similar. By adding the corner functions to the models, a gain in accuracy is obtained, which is similar for the homogeneous cases. For the Robin-Robin boundary conditions, also a gain is obtained, but not as large as for the other cases. At $800 \mathrm{~Hz}$ the results differ more due to the fact that the fields are more complicated, and the influence of singularity depends on the total setting and the field. The convergence of the Dirichlet-Neumann case without corner function is clearly the worst. By adding corner functions to the four models, again a large gain in accuracy is obtained. Similar to the results at $400 \mathrm{~Hz}$, the gain is smallest for the Robin-Robin case. At $800 \mathrm{~Hz}$ the curve of WBM CF DN seems to stagnate. More detail is given in the next study.

\subsubsection{Logarithmic singularities}

As explained in section 4.3, logarithmic singularities can be expected for some specific cases when considering the L-shape problem. As shown in Table 2, this is the case for the combination of a Dirichlet and a non-homogeneous Neumann boundary conditions and an angle of $270^{\circ}$. Logarithmic singularities cannot be taken into account, but the corner function due to homogeneous Dirichlet-Neumann boundary conditions can be added to the wave function set. This type of singularity $\left(\sim O\left(r^{-2 / 3}\right)\right)$ is more severe than the logarithmic singularity $(\sim O(\ln (r)))$. This study evaluates the effect of the presence of a logarithmic singularity on the accuracy of the WBM and three Dirichlet-Neumann cases are compared. The details are given in Table 7. The first case applies a non-homogeneous Neumann boundary 


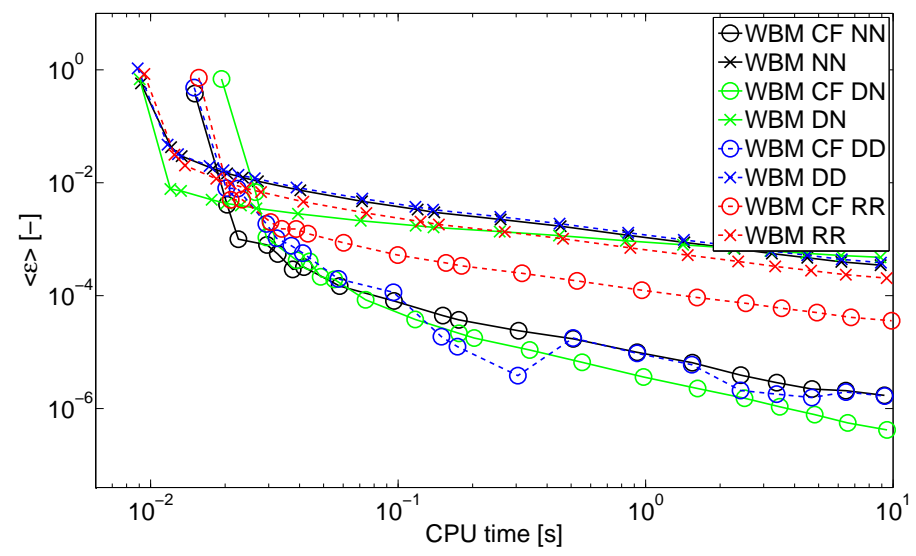

(a) $420 \mathrm{~Hz}$

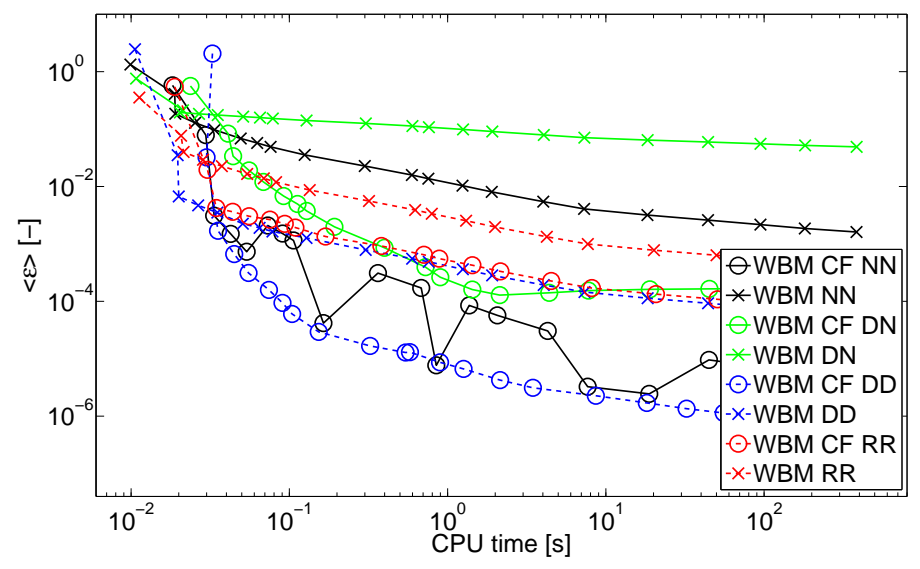

(b) $800 \mathrm{~Hz}$

Figure 16: Convergence curves of the WBM for the L-shape problem applying different boundary conditions near the singular corner at two different frequencies.

condition, $\bar{v}_{n}=1 \mathrm{~m} / \mathrm{s}$, adjacent to the singular corner and leads to a logarithmic singularity. The second case is one of the cases studied in the previous example: homogeneous boundary conditions are applied and the problem domain is excited with an acoustic point source. In the last case no point source is present and the problem domain is excited with a normal velocity $\bar{v}_{n}=1 \mathrm{~m} / \mathrm{s}$ on $\Gamma_{3}$. For those three cases, the same corner function

$$
\Psi_{c_{1}}^{C F}=(\sin (1 / 3 \theta)+\cos (1 / 3 \theta)) J_{1 / 3}(k r),
$$

according to equation (33), is added to the wave function sets of the three subdomains.

Figure 17 shows the convergence curves at $420 \mathrm{~Hz}$ and $800 \mathrm{~Hz}$ respectively of the three different cases. The FE reference models indicated by DN $\mathrm{H} \log$, DN NH and DN H in Table 4 are used. Also the convergence curves of the quadratic adaptively refined FE meshes are shown here, to be able to explain the behaviour of the WB convergence curves. At $420 \mathrm{~Hz}$ a similar gain is obtained for the three WB models by adding the corner function. However, the model containing the logarithmic singularity clearly performs worst. The results at $800 \mathrm{~Hz}$ are different. Again, by 


\begin{tabular}{ll}
\hline Case & excitation \\
\hline $\mathrm{DN} \log$ & $\bar{v}_{n}=1 \mathrm{~m} / \mathrm{s}$ on $\Gamma_{2}$ \\
$\mathrm{DN}$ & point source with $q=1 \mathrm{~Pa}$ at $\mathrm{Q}$ \\
$\mathrm{DN} \mathrm{v}$ & $\bar{v}_{n}=1 \mathrm{~m} / \mathrm{s}$ on $\Gamma_{3}$ \\
\hline
\end{tabular}

Table 7: Three different Dirichlet-Neumann singular cases.

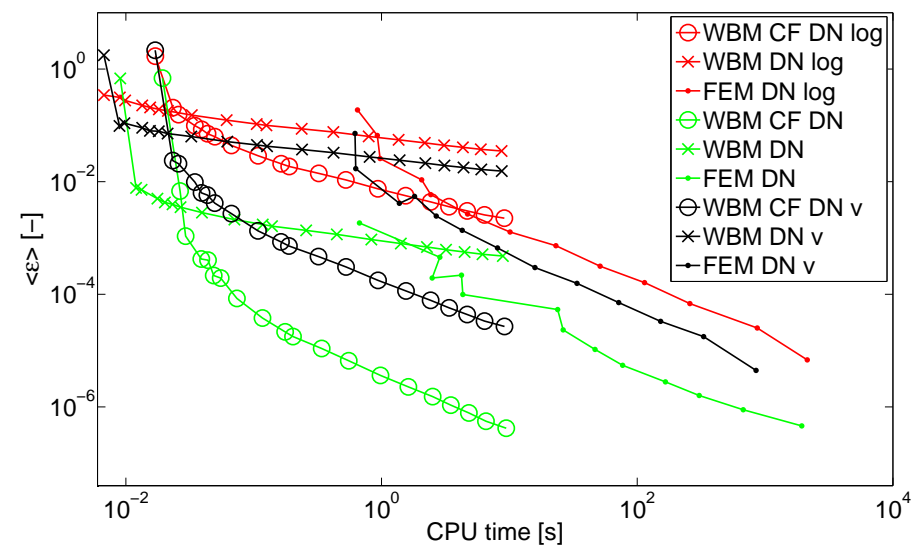

(a) $420 \mathrm{~Hz}$

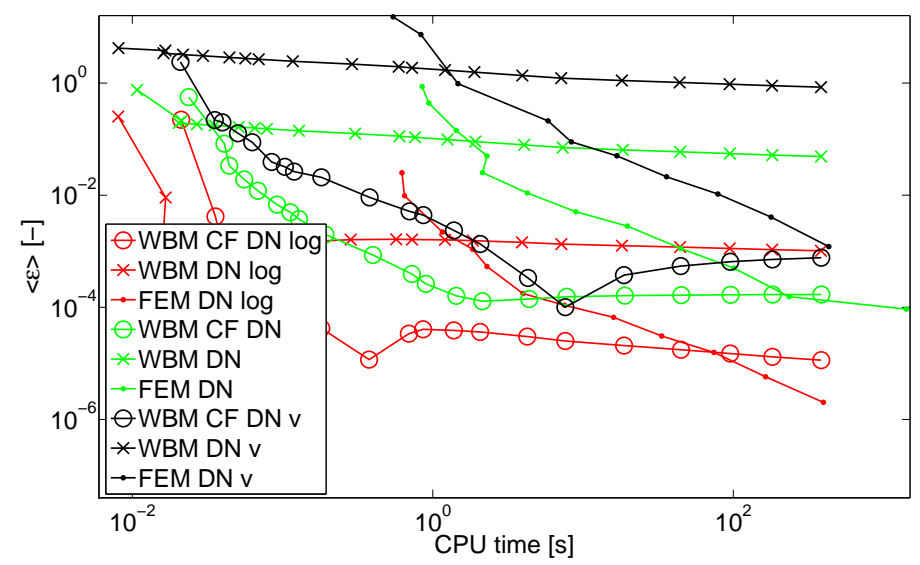

(b) $800 \mathrm{~Hz}$

Figure 17: Convergence curves of the WBM for the Lshape with an increasing number of wave functions at different frequencies.

adding the corner function, a clear improvement is obtained, but in this case the results of the case containing the logarithmic singularity are much improved. The FE results behave similarly as the WB results. It is difficult to predict a priori how easily the response field can be approximated. This in influenced by the geometry, the excitation, the imposed boundary conditions and the frequency of interest. However, adding the corner function always has a very positive influence on the convergence behaviour. Since the singularity is quite severe as compared to DirichletDirichlet or Neumann-Neumann boundary conditions and the wavelengths shorten at $800 \mathrm{~Hz}$, also the FE results converge more slowly at $800 \mathrm{~Hz}$. The WB results stagnate at the accuracy of the finest FE model which explains also 
the behaviour within previous example.

\subsection{Star-shaped scattering problem}

The last example studies the scattering of an incident plane wave on a star-shaped rigid scatterer as shown in Figure 18(a). It verifies if the accuracy of unbounded problems containing singularities can also be improved by adding corner functions. The polygon, with corner points measuring $1 \mathrm{~m}$ from its center, is enclosed by a truncation circle with radius $1.25 \mathrm{~m}$. Rigid boundary conditions are imposed on $\Gamma_{b}$. The plane wave is incident at an angle of $30^{\circ}$ and with an amplitude of 1Pa. Figure 18(b) shows the WB subdivision into four bounded and one unbounded subdomain. The smallest circumscribing bounding boxes of the four bounded subdomains are indicated by thin dashed lines. Each bounding box has dimensions $L_{x}^{(\alpha)}=L_{y}^{(\alpha)}=1.25 \mathrm{~m}$. The problem domain contains four singular corners, which are also indicated in the figure.

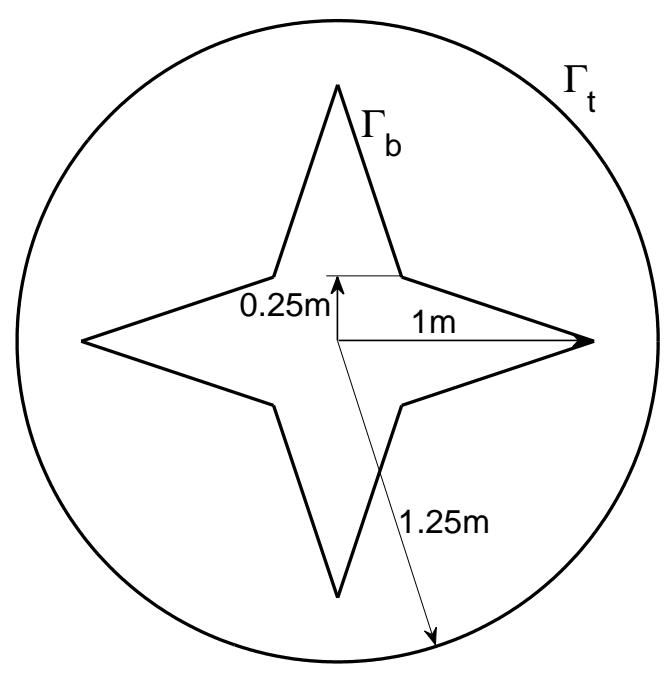

(a) Problem description

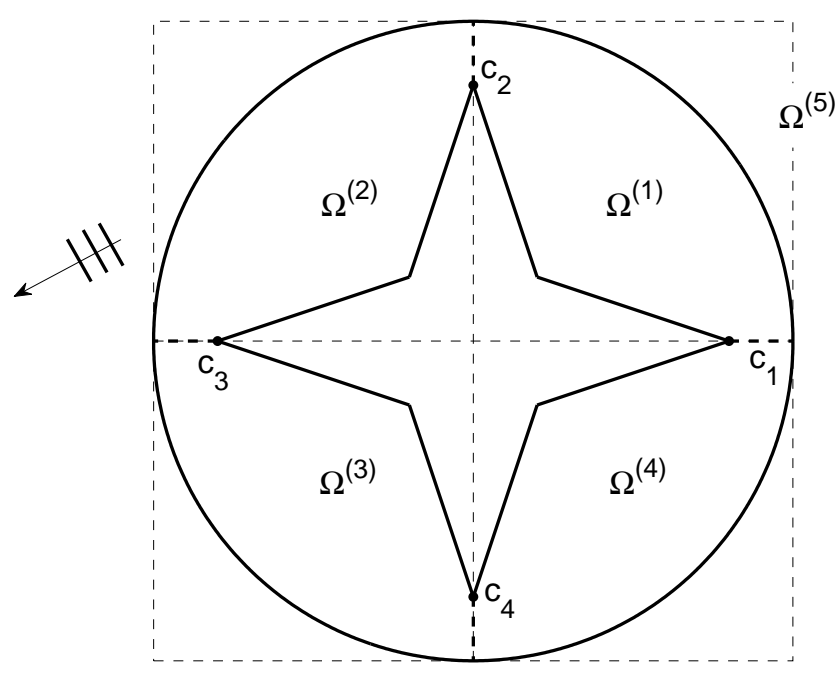

(b) WB subdomains and singular corners

Figure 18: Star-shape scattering problem description

The singular corners are located within the truncation circle which is necessary, as described within section 4.5. For each of the corners $c_{i}$ the angle $\alpha=2 \pi-2 \arctan (1 / 3)>\pi$, giving one eigenvalue $\lambda_{1}^{*}=\pi / \alpha$ leading to a singularity according to equation (32). Consequently, four corner functions

$$
\Psi_{c_{i}}^{C F}=\sin \left(\lambda_{1}^{*} \theta\right) J_{\lambda_{1}^{*}}(k r),
$$

defined in four local coordinate systems attached to each the singular corners, are added to the wave function expansions of the two adjacent subdomains.

Figure 19 shows the real part of the scattered pressure field at $1000 \mathrm{~Hz}$, calculated with the WBM CF. The complex geometry gives rise to interference and diffraction patterns. The results are obtained applying a truncation factor $N=4$. The pressure field is continuous, also in the vicinity of the singular corner points. 


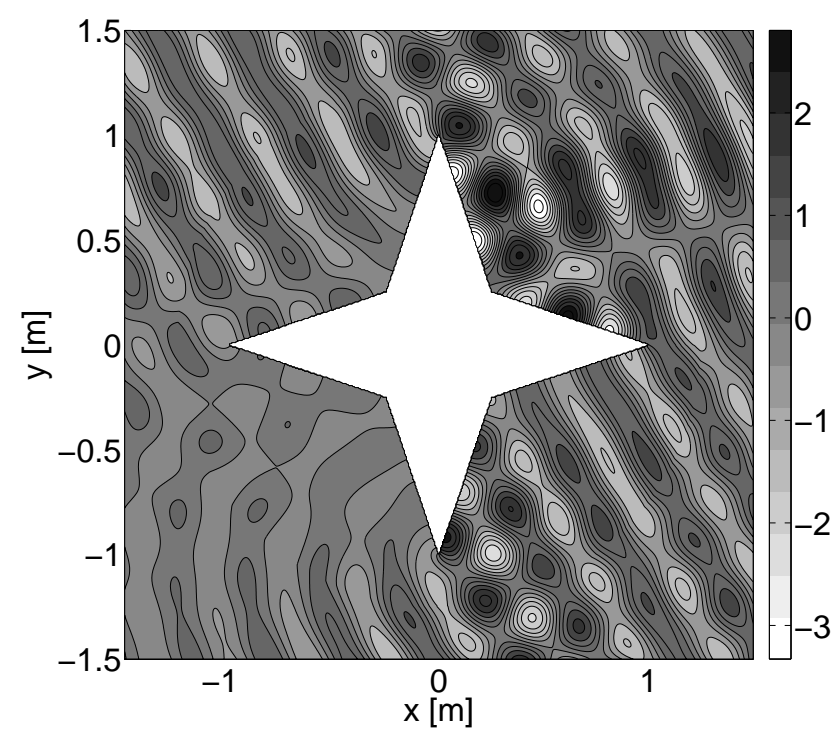

Figure 19: Real part of the scattered pressure field at $1000 \mathrm{~Hz}$, calculated with the WBM CF.

Also convergence curves are calculated for this geometry. A BE reference is obtained using a very fine mesh with $0.25 \mathrm{~mm}$ element length. The coarsest and finest WB models are obtained by applying a truncation factor $N=0.5$ and $N=6$, respectively. In between these models, $N$ is linearly increased with steps of 0.5 . The WBM CF models always contain four additional corner functions as compared to the models in the table. The results are averaged over approximately 1300 response points distributed over the five subdomains and the shown relative error is again calculated applying equation (38). Figure 20 shows the results obtained by the WBM and the WBM CF at respectively 500,1000 and $1500 \mathrm{~Hz}$. Also for this more complex geometry, which results in short-wavelength waves often travelling almost tangent to $\Gamma_{t}$, a very good accuracy is obtained by adding the four corner functions. An improvement of approximately two orders of magnitude in prediction accuracy is thus obtained similar to bounded acoustic problems.

\section{Conclusions}

The WBM is a Trefftz based prediction technique which aims to be an alternative to element-based techniques. However, the accuracy of the WB predictions are hampered when singularities are present at the corners of the acoustic domains. This paper discusses criteria to determine whether singularities are present and proposes the use of special purpose enrichment functions in the wave function expansion set to accurately capture this singular behaviour. These so-called corner functions are obtained by applying an asymptotic analysis to find solutions of the 2D Helmholtz equation which also fulfil the boundary conditions and regularity conditions at the corner tip. For all homogeneous combinations of boundary conditions, exact analytical solutions are found and can be added to the wave function expansion. For other combinations of boundary conditions, solutions of the Laplace equation can be used to verify 


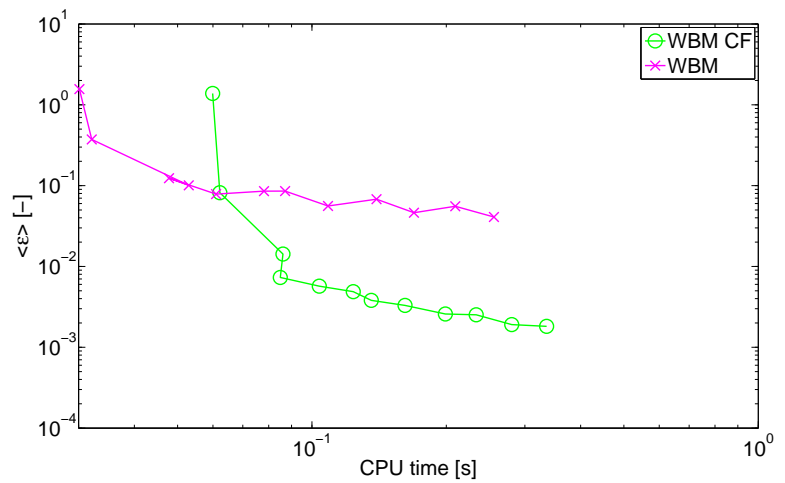

(a) $500 \mathrm{~Hz}$

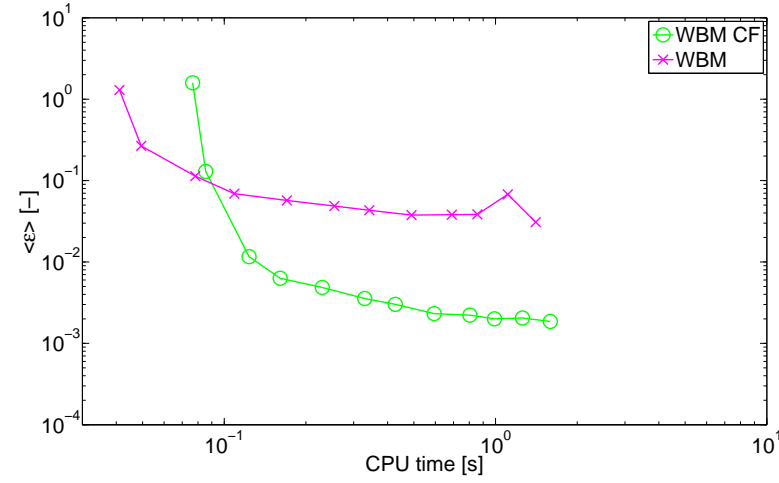

(b) $1000 \mathrm{~Hz}$

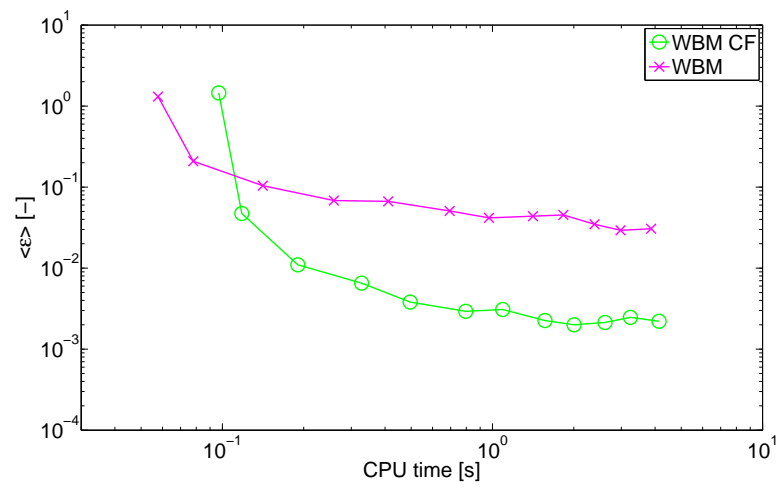

(c) $1500 \mathrm{~Hz}$

Figure 20: Convergence curves for the star-shape problem at three frequencies for the WBM and the WBM CF.

if additional singularities are present. Three numerical examples applying different combinations of boundary conditions show the beneficial effect of adding the enrichment functions, for bounded as well as for unbounded problems. The convergence rate increases at a small additional cost and for the shown examples 1 to 2 orders of accuracy are gained. The research on this topic will be continued by extending the approach to multi-material acoustic problems containing singularities. The obtained results in this paper are also very useful for the further study and interpretation of singularities existing in poroelastic materials, which consist of both a solid and a fluid phase. Singularities can exist in both phases and the results obtained within this paper can be applied to tackle part of them.

\section{Acknowledgements}

Elke Deckers is a Doctoral Fellow of the Fund for Scientific Research-Flanders (F.W.O.), Belgium. Furthermore, the authors gratefully acknowledge the European Commission for their support of the ITN Marie Curie project GA214909 "MIDFREQUENCY - CAE Methodologies for Mid-Frequency Analysis in Vibration and Acoustics". The IWT Flanders within the ASTRA project, the Fund for Scientific Research - Flanders (F.W.O.), and the Research Fund KU Leuven are also gratefully acknowledged for their support. 


\section{Appendix A. Derivation of the solutions of the acoustic infinite wedge problem}

Assuming a separable function for the pressure field $p(r, \theta)$ in polar coordinates leads to the following homogeneous solution of the Helmholtz equation [52]:

$$
p(r, \theta)=\sum_{b=1}^{\infty}\left[\cos \left(\lambda_{b} \theta\right) R_{\lambda_{b}}(k r)+\sin \left(\lambda_{b}^{*} \theta\right) R_{\lambda_{b}^{*}}(k r)\right],
$$

where

$$
R_{\lambda}(z)=A_{\lambda} J_{\lambda}(z)+B_{\lambda} Y_{\lambda}(z)
$$

with $A_{\lambda}$ and $B_{\lambda}$ constants of integration, $J_{\lambda}(z)$ and $Y_{\lambda}(z)$ the ordinary Bessel functions of the first and the second kind and $\lambda=\lambda_{b}$ or $\lambda_{b}^{*}$ the eigenvalues. The eigenvalues $\lambda_{b}$ are associated with symmetric eigenfunctions with respect to the axis $\theta=0$ and the eigenvalues $\lambda_{b}^{*}$ are associated with anti-symmetric eigenfunctions. By applying the boundary conditions at $\theta= \pm \alpha / 2$, one obtains a system of equations which can be solved for the unknown integration constants:

$$
[\mathbf{A}]\{\mathbf{c}\}=\{\mathbf{0}\},
$$

with [A] the coefficient matrix and $\{\mathbf{c}\}$ the vector of unknown constants of integration, $A_{\lambda}$ and $B_{\lambda}$. Since trivial solutions should be eliminated, the determinant of the coefficient matrix must be zero:

$$
\mathcal{D}=\operatorname{det}[\mathbf{A}]=0,
$$

and the eigenvalues $\lambda_{b}$ and $\lambda_{b}^{*}$, associated with symmetric and anti-symmetric eigenfunctions, are the solutions of this characteristic equation. Since it is assumed that the boundary conditions at infinity have no influence on the singular behaviour at the corner, they are discarded. The absence of requirements at infinity results in solutions of (A.3) which are non-unique. Since the main purpose of this analysis is the characterisation of all possible singularities at the corner point, this is not a problem. In a configuration with a finite extent, locally containing the configuration studied above, the boundary conditions applied to the other boundaries will make the solution unique. In the WB approach, this is done by applying the weighted residual formulation.

Regularity conditions have to be imposed on the corner tip $(r=0)$, assuring that the pressure field remains finite:

$$
|p(0, \theta)|<\infty .
$$

Since the Bessel functions of the second kind $Y_{\lambda}(r)$ are inherently unbounded at $r=0$ [53], all integration constants $B_{\lambda}$ have to be zero. Since Bessel functions of the first kind can be expanded using a power series [53]:

$$
J_{\lambda}(z)=\sum_{k=0}^{\infty} \frac{(-1)^{k}}{k ! \Gamma(\lambda+k+1)}\left(\frac{z}{2}\right)^{2 k+\lambda},
$$

the remaining expressions are finite if all the eigenvalues are equal or larger than 0 . Taking this into account, the eigenfunctions become:

$$
R_{\lambda}=A_{\lambda} J_{\lambda}(k r) \text { with } \lambda \geq 0,
$$


yielding the following admissible pressure fields:

$$
p(r, \theta)=\sum_{b=1}^{\infty}\left[A_{\lambda_{b}} \cos \left(\lambda_{b} \theta\right) J_{\lambda_{b}}(k r)+A_{\lambda_{b}^{*}} \sin \left(\lambda_{b}^{*} \theta\right) J_{\lambda_{b}^{*}}(k r)\right] .
$$

The velocity field components can be obtained using the following expressions:

$$
\begin{aligned}
& v_{r}(r, \theta)=\frac{j}{\rho \omega} \frac{\partial p}{\partial r}, \\
& v_{\theta}(r, \theta)=\frac{j}{\rho \omega} \frac{1}{r} \frac{\partial p}{\partial \theta} .
\end{aligned}
$$

When substituting the admissible pressure fields (A.8), this yields:

$$
\begin{aligned}
& v_{r}(r, \theta)=\frac{j}{\rho \omega} \sum_{b=1}^{\infty}\left[\frac{A_{\lambda_{b}} k}{2} \cos \left(\lambda_{b} \theta\right)\left(J_{\lambda_{b}-1}(k r)-J_{\lambda_{b}+1}(k r)\right)+\frac{A_{\lambda_{b}^{*} k}}{2} \sin \left(\lambda_{b}^{*} \theta\right)\left(J_{\lambda_{b}^{*}-1}(k r)-J_{\lambda_{b}^{*}+1}(k r)\right)\right], \\
& v_{\theta}(r, \theta)=\frac{j}{\rho \omega} \frac{1}{r} \sum_{b=1}^{\infty}\left[-A_{\lambda_{b}} \lambda_{b} \sin \left(\lambda_{b} \theta\right) J_{\lambda_{b}}(k r)+A_{\lambda_{b}^{*}} \lambda_{b}^{*} \cos \left(\lambda_{b}^{*} \theta\right) J_{\lambda_{b}^{*}}(k r)\right] .
\end{aligned}
$$

Using the power series of Bessel functions of the first kind, equation (A.6), these expressions tend to infinity at $r \rightarrow 0$ if $0<\lambda<1$. The eigenvalues $\lambda$ depend on $\alpha$ and the applied boundary conditions on both sides of the wedge. In the case $0<\lambda<1$, a singularity can exist in the velocity field and the order of the singularity is $\lambda-1$.

\section{References}

[1] O. C. Zienkiewicz, R. L. Taylor, and J. Z. Zhu. The Finite Element Method - Vol. 1: Its Basis E Fundamentals. Butterworth-Heinemann, 6th edition, 2005

[2] O. Von Estorff. Boundary Elements in Acoustics: Advances and Applications. WITpress, 2000.

[3] Z.C. Li and T.T. Lu. Singularities and Treatment of Elliptic Boundary Value Problems. Math. Comput. Modelling, 31:97-145, 2000.

[4] I. Babuška and H.S. Oh. The p-version of the finite element method for domains with corners and for infinite domains. Numer. Methods Partial Differential Equations, 6:371-392, 1990.

[5] T. Apel, A.-M. Sändig, and J.R. Whiteman. Graded Mesh Refinement and Error Estimates for Finite Element Solutions of Elliptic Boundary Value Problems in Non-Smooth Domains. Math. Methods Appl. Sci., 19:63-85, 1996.

[6] T. Apel and S. Nicaise. The Finite Element Method with Anisotropic Mesh Grading for Elliptic Problems in Domains with Corners and Edges. Math. Methods Appl. Sci., 21:219-549, 1998.

[7] M. Maischak, P. Mund, and E.P. Stephan. Adaptive multilevel BEM for acoustic scattering. Comput. Methods Appl. Mech. Engrg., 150:351$367,1997$.

[8] J. Jirousek and A. Venkatesh. A new fe approach for adaptive reliability assurance. Computer $\mathcal{G}$ Structures, 37:217-230, 1990.

[9] L.G. Olson, G.C. Georigiou, and W. W. Schultz. An efficient finite element method for treating singularities in Laplace's equation. J. Comput. Phys., 96:391-410, 1991.

[10] X. Wu and H. Han. A finite-element method for Laplace- and Helmholtz-type boundary value problems with singularities. SIAM J. Numer. Anal., 34:1037-1050, 1997.

[11] T.R. Lucas and H.S. Oh. The Method of Auxiliary Mapping for the Finite Element Solutions of Elliptic Problems Containing Singularities. J. Comp. Phys., 108:327-342, 1993.

[12] D. Givoli, L. Rivkin, and J.B. Keller. A finite element method for domains with corners. Int. J. Numer. Methods Engrg., 35:1329-1345, 1990. 
[13] W. Cai, H.C. Lee, and H.S. Oh. Coupling of Spectral Methods and the p-version of the Finite Element Method for Elliptic Boundary Value Problems Containing Singularities. J. Comp. Phys., 108:314-326, 1993.

[14] J.T. Chen and K.H. Chen. Dual integral formulation for determining the acoustic modes of a two-dimenstional cavity with a degenerate boundary. Engineering Anaysis with Boundary Elements, 21:105-116, 1998.

[15] J.T. Chen, M.T. Liang, I.L. Chen, S.W. Chyuan, and K.H. Chen. Dual boundary element analysis of wave scattering from singularities. Wave Motion, 30:367-381, 1999.

[16] L. Marin, D. Lesnic, and V. Mantič. Treatment of singularities in Helmholtz-type equations using the boundary element method. J. Sound Vibr., 278:39-62, 2004.

[17] I. Harari and T.J.R. Hughes. A cost comparison of boundary element and finite element methods for problems of time-harmonic acoustics. Computer Methods in Applied Mechanics and Engineering, 97:77-102, 1992.

[18] C. Farhat, I. Harari, and L.P. Franca. The discontinuous enrichment method. Computer Methods in Applied Mechanics and Engineering, 190:6455-6479, 2001.

[19] R. Tezaur, L. Zhang, and C. Farhat. A discontinuous enrichment method for capturing evanescent waves in multiscale fluid and fluid/solid problems. Computer Methods in Applied Mechanics and Engineering, 197:1680-1698, 2008.

[20] E. Trefftz. Ein Gegenstück zum Ritzschen Verfahren. In Proceedings of the $2^{\text {nd }}$ International Congress on Applied Mechanics, Zurich, Switzerland, pages 131-137, 1926.

[21] B. Pluymers, B. Van Hal, D. Vandepitte, and W. Desmet. Trefftz-based methods for time-harmonic acoustics. Archives of Computational Methods in Engineering, 14(4):343-381, 2007.

[22] H. Riou, P. Ladevèze, and B. Sourcis. The multiscale VTCR approach applied to acoustic problems. J. Comput. Acoustics, 16:487-505, 2008.

[23] T. Huttunen, P. Gamallo, and R.J. Astley. Comparison of two wave element methods for the Helmholtz problem. Commun. Numer. Meth. Engng., 25:35-52, 2009.

[24] P. Gamallo and R.J. Astley. A comparison of two Trefftz-type methods: The ultraweak variational formulation and the least-squares method, for solving shortwave 2-D Helmholtz problems. Int. J. Numer. Meth. Engng., 71:406-432, 2007.

[25] O. Cessenat and B. Depres. Application of an ultra weak variational formulation of elliptic PDEs to the two-dimensional Helmholtz problem. SIAM J. Numer. ANAL., 35:255-299, 1998.

[26] Z.C. Li. The Trefftz method for the Helmholtz equation with degeneracy. Appl. Numer. Math., 58:131-159, 2008.

[27] P.R.S. Antunes and S.S. Valtchev. A meshfree numerical method for acoustic wave propagation problems in planar domains with corners and cracks. J. Comput. Appl. Math., 234:2646-1662, 2010.

[28] L. Marin. Treatment of singularities in the method of fundamental solutions for two-dimensional Helmholtz-type equations. Appl. Math. Model., 34:1615-1633, 2010.

[29] M. Stojek. Least-squares Trefftz-type elements for the Helmholtz equation. Int. J. Numer. Methods Engng., 41:831-849, 1998.

[30] A.H. Barnett and T. Betcke. An exponentially convergent nonpolynomial finite element method for time-harmonic scattering from polygnons. SIAM J. Sci. Comput., 32:1417-1441, 2010.

[31] W. Desmet. A wave based prediction technique for coupled vibro-acoustic analysis. KULeuven, division PMA, PhD. thesis 98D12, 1998.

[32] B. Van Genechten, K. Vergote, D. Vandepitte, and W. Desmet. A multi-level wave based numerical modelling framework for the steady-state dynamic analysis of bounded Helmholtz problems with multiple inclusions. Comput. Methods Appl. Mech. Engrg., 199:1881-1905, 2010.

[33] B. Van Genechten, B. Bergen, D. Vandepitte, and W. Desmet. A Trefftz-based numerical modelling framework for Helmholtz problems with complex multiple scatterer configurations. Journal of Computational Physics, 229(18):6623-6643, 2010.

[34] B. Bergen, B. Van Genechten, D. Vandepitte, and W. Desmet. An efficient Trefftz-based method for three-dimensional Helmholtz problems in unbounded domains. Computer Modeling in Engineering E Sciences, 61(2):155-175, 2010.

[35] B. Bergen, B. Pluymers, B. Van Genechten, D. Vandepitte, and W. Desmet. A Trefftz based method for solving Helmholtz problems in semi-infinite domains. Engineering Analysis with Boundary Elements, 36:30-38, 2012. 
[36] C. Vanmaele, D. Vandepitte, and W. Desmet. An efficient wave based prediction technique for plate bending vibrations. Comput. Methods Appl. Mech. Engrg., 196:3178-3189, 2007.

[37] C. Vanmaele, D. Vandepitte, and W. Desmet. An efficient wave based prediction technique for dynamic plate bending problems with corner stress singularities. Comput. Methods Appl. Mech. Engrg., 198:2227-2245, 2009.

[38] E. Deckers, N.-E. Hörlin, D. Vandepitte, and W. Desmet. A Wave Based Method for the efficient solution of the 2D poroelastic Biot equations. Comput. Methods Appl. Mech. Engrg., 201-204:245-262, 2012.

[39] E. Deckers, B. Van Genechten, D. Vandepitte, and W. Desmet. Efficient treatment of stress singularities in poroelastic wave based models using special purpose enrichment functions. Comput. Struct., 89:1117-1130, 2011.

[40] B. Van Genechten, D. Vandepitte, and W. Desmet. A direct hybrid finite element - Wave based modelling technique for efficient coupled vibro-acoustic analysis. Comput. Methods Appl. Mech. Engrg., 200:742-764, 2011.

[41] K. Vergote, B. Van Genechten, D. Vandepitte, and W. Desmet. On the analysis of vibro-acoustic systems in the mid-frequency range using a hybrid deterministic-statistical approach. Comput. Struct., 89:868-877, 2011.

[42] A. Pierce. Acoustics: An Introduction to Its Physical Principles and Applications. McGraw-Hill series in mechanical engineering, McGrawHill, 1981.

[43] D. Colton and R. Kress. Inverse acoustic and electromagnetic scattering theory. Springer-Verlag, Berlin, Heidelbert, New York, 2nd edition, 1998.

[44] B. Pluymers. Wave based modelling methods for steady-state vibro-acoustics. KULeuven, division PMA, PhD. thesis 2006D04, 2006.

[45] B. Bergen. Wave Based modelling techniques for unbounded acoustic problems. KULeuven, division PMA, PhD. thesis 2011D07, 2011.

[46] I. Herrera. Boundary Methods: an Algebraic Theory. Pitman Adv. Publ. Program, London, 1984.

[47] E. Deckers, B. Drofmans, B. Van Genechten, B. Bergen, D. Vandepitte, and W. Desmet. Spline-based boundaries: a first step towards generic geometric domain descriptions for effcient mid-frequency acoustic analysis using the Wave Based Method. J. Comput. Appl. Math., 235:2679-2693, 2011.

[48] R.F. Millar. Singularities of two-dimensional exterior solutions of the Helmholtz equation. Proc. Camb. Phil. Soc., 69:175-188, 1971.

[49] G.B. Sinclair. Stress singularities in classical elasticity - I: Removal, interpretation and analysis. Applied Mechanics Reviews, 57:251-197, 1993.

[50] K. Wolsson. Linear dependence of a function set of $m$ variables with vanishing generalized Wronskians. Linear Algebra and its Applications, 117:73-80, 1989

[51] H. Motz. The treatment of singularities of partial differential equations by relaxation methods. Quart. App. Math., 4:371-377, 1947.

[52] P. Moon and D.E. Spencer. Field Theory Handbook Including Coordinate Systems, Differential Equations And Their Solutions. Prentice Hall, New Jersey, 1961.

[53] M. Abramowitz and A. Stegun. Handbook of mathematical functions with formulas, graphs and mathematical tables. Dover publications, Inc., New York, 1970. 